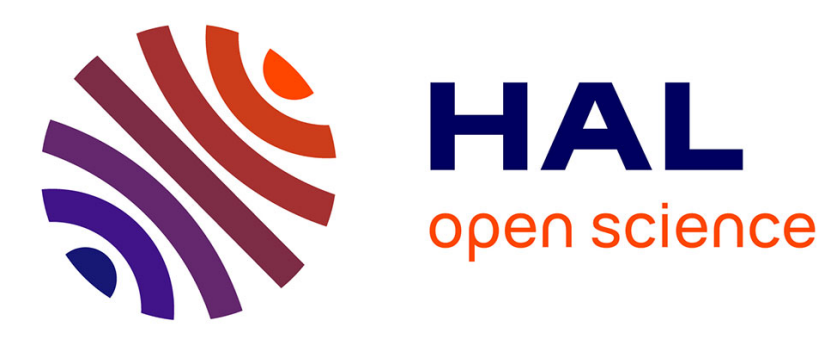

\title{
A Globally Convergent Frequency Estimator
}

Liu Hsu, Romeo Ortega, G. Damm

\section{To cite this version:}

Liu Hsu, Romeo Ortega, G. Damm. A Globally Convergent Frequency Estimator. IEEE Transactions on Automatic Control, 1999, 10.1109/9.754808 . hal-02936817

\section{HAL Id: hal-02936817 https://hal.science/hal-02936817}

Submitted on 11 Sep 2020

HAL is a multi-disciplinary open access archive for the deposit and dissemination of scientific research documents, whether they are published or not. The documents may come from teaching and research institutions in France or abroad, or from public or private research centers.
L'archive ouverte pluridisciplinaire HAL, est destinée au dépôt et à la diffusion de documents scientifiques de niveau recherche, publiés ou non, émanant des établissements d'enseignement et de recherche français ou étrangers, des laboratoires publics ou privés. 


\title{
A globally convergent frequency estimator
}

\author{
${ }^{\dagger}$ Liu Hsu, ${ }^{\ddagger}$ Romeo Ortega ${ }^{*}$ and ${ }^{\ddagger}$ Gilney Damm
}

\author{
${ }^{\dagger}$ Electrical Engineering \\ COPPE/UFRJ \\ P.O. Box 68504, 21945/970 \\ Rio de Janeiro, BRAZIL \\ liu@coep.ufrj.br
}

\author{
$\ddagger$ Laboratoire des Signaux et Systemes \\ CNRS-SUPELEC \\ Plateau de Moulon, 91192 \\ Gif sur Yvette, FRANCE \\ [rortega, damm] @lss.supelec.fr
}

June 30, 1999

\begin{abstract}
On-line estimation of the frequency of a sinusoidal signal is a classical problem in systems theory that has many practical applications. In this paper we provide a solution to the problem of ensuring a globally convergent estimation. More specifically, we propose a new adaptive notch filter whose dynamic equations exhibit the following remarkable features: i) all signals are globally bounded and the estimated frequency is asymptotically correct for all initial conditions and all frequency values; ii) we obtain a simple tuning procedure for the estimator design parameters, which trades-off the adaptation tracking capabilities with noise sensitivity, ensuring (exponential) stability of the desired orbit; iii) transient performance is considerably enhanced, even for small or large frequencies, as witnessed by extensive simulations. To reveal some of the stability-instability mechanisms of the existing algorithms and motivate our modifications we make appeal to a novel nonlinear (state-dependent) time scaling. The main advantage of working in the new time scale is that we remove the coupling between the parameter update law and the filter itself, decomposing the system into a feedback form where the required modifications to ensure stability become apparent. Even though we limit our attention here to the simplest case of a single constant frequency without noise the algorithm is able to track time-varying frequencies, preserves local stability in the presence of multiple sinusoids, and is robust with respect to noise.
\end{abstract}

Keywords: frequency estimation, adaptive notch filter, noise cancellation, stability analysis, nonlinear systems, adaptive signal processing.

\section{Introduction}

We are interested here in the problem of continuous-time on-line estimation of the frequency $\theta_{*}>0$ of a pure sinusoidal signal

$$
n=k \sin \left(\theta_{*} t\right)
$$

where $n$ is measurable, and its amplitude, $k>0$, is also unknown. ${ }^{1}$ Frequency estimation is a fundamental question in systems theory that has many practical applications, for instance in active noise and vibration control [4] in helicopters [9], disk drives [2] and magnetic bearings [10]. It is also a challenging theoretical problem (even in the case when noise is absent) since the nonlinear dependence on the unknown frequency stymies the application of standard well known techniques. For instance, if it is expressed using a statespace realization of the sinusoid, we are confronted with a problem of simultaneous estimation of the state and the parameter, which is a well-known open problem in systems theory. Although in discrete-time it is possible to obtain a linear parametrization, hence side-stepping the aforementioned obstacle, the critical

\footnotetext{
* Author to whom all correspondence should be addressed.

${ }^{1}$ The estimators considered here are in fact applicable for the more general case $n=k \sin \left(\theta^{*} t+\phi\right)$, where $\phi$ is a constant but unkown phase.
} 
dependence of the estimate on the sampling time (particularly for frequencies close to 0 or $\pi$ ) makes this formulation inadequate in a global context. Also, it is clear that a direct off-line solution is possible, but we are looking here for on-line implementations that attenuate (via averaging) the noise effects and are able to track time-varying frequencies.

There exists several algorithms to estimate on-line the frequency of a sinusoid; for a detailed review see the classical paper [12], or the more recent work of [8]. Probably, the first adaptive solution was the line enhancer of [29], later analyzed in [28], which amounts to the implementation of an adaptive $k$-step ahead predictor with a finite impulse response (all zero) filter. When suitably tuned, the scheme converges to a polynomial with zeros on the unit circle, having angular locations corresponding to the signal frequencies. An improved formulation of this scheme was later reported in [21] and [22]. Finite impulse response filters proved deficient in recuperating the sinusoid since sharp cutoff characteristics are needed. This motivated the consideration of adaptive infinite impulse response filters (with poles and zeros) in [7]. The constraints imposed on notch filters yield simple relations between the poles and the zeros. Without imposing such constraints serious problems of numerical instability arise. The one-parameter-per-sinusoid notch model, which advantageously exploited these constraints, was first proposed in [19], and has since been studied by many other authors, see the references in [24] and [1].

To the best of our knowledge, in spite of this intensive research, the basic problem of designing a globally convergent estimator remained open. In this paper we provide a solution to this problem. More specifically the main contribution in this paper is the development of a continuous-time on-line frequency estimator with the following remarkable features:

- all signals are globally bounded and, for sufficiently slow adaptation, the estimated frequency is asymptotically correct for all initial conditions and all frequency values;

- we propose a simple tuning procedure for the design parameters, which trades-off the adaptation tracking capabilities with noise sensitivity, always ensuring (exponential) stability;

- the estimated frequency is restricted to take nonegative values, without the introduction of parameter projections;

- transient performance is considerably enhanced, even for small and large frequencies, as witnessed by extensive simulations.

Our frequency estimator is an adaptive notch filter (ANF), which takes off from the ANF proposed in [23], later adapted for continuous-time in the interesting paper [2]. As pointed out in the latter, the dynamic equations that describe ANF's, although of low order, are extremely complicated. Their stability analysis has been limited to the application of local averaging techniques under the assumption of slow adaptation. Even though the predictions made with this analysis are quite accurate (at least under slow adaptation and close to the equilibrium), our concern to obtain global stability motivates us to further study the underlying stability-instability mechanisms of this ANF. This is done in section 2 of our paper, where we present Regalia's ANF and, -in the spirit of [25]-, we prove the existence of (a family of) slow adaptation integral manifolds. In particular, we explicitely define the frozen parameter integral manifold, which contains the desired orbit, and prove that the motion on this manifold is stable. More importantly, we show that for small frequencies adaptation speeds-up triggering instability, while, on the other hand, for large frequencies adaptation slows down and performance is degraded.

To avoid the two latter undesirable phenomena we propose in section 3 the simple, but essential, modification of scaling the ANF's forcing term. We also show that scaling does not affect stability under slow adaptation. It modifies, though, the shape of the frozen parameter integral manifold. An additional beneficial effect of scaling is that it ensures the estimate is always positive. (Besides its obvious practical appeal, this feature is important in the sequel to insure global existence of a nonlinear time-scaling introduced to decouple the filter from the estimator).

The scaled ANF is, however, not globally stable, solutions may escape to infinity for sufficiently large initial conditions. To further proceed with our goal of obtaining a globally convergent scheme we introduce in section 4 a novel nonlinear (state-dependent) time scaling. ${ }^{2}$ The main advantage of working in the new time

\footnotetext{
${ }^{2}$ See [20] for an application of time-scaling to the analysis of an adaptive system and some illustrative examples.
} 
scale is that we "decouple" the parameter update law from the filter itself, obtaining a representation of the ANF as a feedback interconnection of a stable linear time-invariant (LTI) system with a "nonlinear gain". It turns out that this gain can be bounded with the introduction of a suitable normalization factor in the estimator, rendering the system amenable for $\mathcal{L}_{\infty}$ small-gain analysis. In this way we obtain an upperbound on the adaptation gain that ensures the filter signals are globally bounded. Our main result is presented in section 5 where we establish global asymptotic convergence of the estimated frequency, for sufficiently slow adaptation speeds, of the proposed scaled and normalized ANF.

ANF's contain two design parameters, the adaptation gain that determines the speed of adaptation, -hence its alertness to track frequency variations-, and the damping coefficient that determines the "depth of the notch", and consequently its noise sensitivity. A further contribution of our paper, which is presented in section 6, is the development of a tuning procedure to trade-off between adaptation alertness and noise sensitivity, preserving (exponential) stability. This is a very delicate task because, as we first show, the ANF may exhibit very complex dynamic behaviour -even close to the steady-state- if the parameters are not properly tuned. In particular we show that the tangent approximation of the dynamics along the desired orbit is described by two coupled Mathieu's equations. Hence the (analytical) characterization in parameter space of the stability-instability boundary is ruled out. Since the frequency and the amplitude of the sinusoid are unknown, our tuning procedure satisfies the sine qua non conditions of being frequency-independent and robust to amplitude uncertainty.

We wrap up the paper with some concluding remarks and further research in section 7 . In particular, we point out that even though we concentrated our attention here in the simplest case of a single constant frequency without noise, in [5] we have shown, -via simulations and some analysis,- that the algorithm is able to track time-varying frequencies, preserves local stability in the face of multiple sinusoids, and is robust vis à vis noise.

Throughout the paper we present simulation results that corroborate our claims. Extensive simulations, further details on the present work and applications to the noise cancelling problem may be found in [5].

\section{The algorithm of Regalia: Stability and instability}

In this section we present Regalia's ANF, prove that it has a unique periodic orbit at the desired frequency, and explain, via an approximate frozen parameter analysis, the mechanism by which it renders this orbit stable for sufficiently slow adaptation. This approximate reasoning is later formalized proving, -in the spirit of [25]-, the existence of (a family of) slow adaptation integral manifolds. In particular, we explicitely define the frozen parameter integral manifold which contains the desired orbit. More importantly, we show that for small frequencies adaptation speeds up triggering instability, while, on the other hand, for large frequencies adaptation slows down and performance is degraded.

\subsection{Motivation}

One popular alternative to solve the frequency estimation problem is to use an ANF [24]. A notch filter is an LTI system whose magnitude response vanishes at a particular point in the $j \omega$ axis, which is called the notch frequency, and whose magnitude response is nearly constant at other points. Excellent approximations are obtained using second-order filters, where to insure that the filter is proper a bandwidth parameter that determines the "notch depth" is added. In this way, feeding $n$ to the notch filter

$$
H_{N}(p)=\frac{p^{2}+\bar{\theta}^{2}}{p^{2}+2 \zeta \bar{\theta} p+\bar{\theta}^{2}}
$$

will provide (up to exponentially decaying exponentials) a zero output when the notch is centered in $\theta_{*}$, i.e. when $\bar{\theta}=\theta_{*}$. It is then reasonable to combine a "time-varying" notch filter with an estimator that adjusts the notch location using the information from the filter output. Since the unknown parameter enters nonlinearly, the question is, of course, how to implement the estimator to guarantee some stability properties? In [23] a very interesting procedure to carry out this task was presented. As opposed to the classical approach of minimizing an output error cost function (which generates local minima [24], [1]) in [23] the estimator is designed to achieve a stable averaged behaviour [14]. 
The equations of Regalia's ANF, later transposed to continuous-time in [2], are given as

$$
\begin{aligned}
\ddot{x}+2 \zeta \theta \dot{x}+\theta^{2} x & =n \\
\dot{\theta} & =-\gamma x(n-2 \zeta \theta \dot{x})
\end{aligned}
$$

where $\theta$ represents the estimated frequency, $\zeta>0$ is the damping coefficient, and $\gamma>0$ determines the adaptation speed ${ }^{3}$. These two design parameters are used to trade-off between adaptation alertness to track frequency variations and noise sensitivity. As will become clear below, the initial conditions of the ANF are typically chosen as $\left(x\left(t_{0}\right), \dot{x}\left(t_{0}\right), \theta\left(t_{0}\right)\right)=\left(\frac{-\hat{k}}{2 \zeta \theta_{0}^{2}}, 0, \theta_{0}\right) \in \mathcal{R}^{3}$, where $\hat{k}$ and $\theta_{0}$ are initial guesses for $k$ and $\theta_{*}$, respectively. ${ }^{4}$

The fact below follows inmediately from the ANF's equations.

\section{Fact 2.1}

The dynamical system (2.1), (2.2) has a unique periodic orbit with constant (and correct) estimated frequency given by ${ }^{5}$

$$
\mathcal{O} \triangleq\left[\begin{array}{c}
\bar{x} \\
\dot{\bar{x}} \\
\bar{\theta}
\end{array}\right]=\left[\begin{array}{c}
\frac{-k}{2 \zeta \theta_{*}^{2}} \cos \left(\theta_{*} t\right) \\
\frac{k}{2 \zeta \theta_{*}} \sin \left(\theta_{*} t\right) \\
\theta_{*}
\end{array}\right]
$$

To provide some insight into the ANF's operation, let us assume that adaptation has frozen. In that case (2.1) reduces to

$$
x=\frac{1}{p^{2}+2 \zeta \bar{\theta} p+\bar{\theta}^{2}} n
$$

with $\bar{\theta}$ constant. Then, the signal driving the adaptation law becomes

$$
x(n-2 \zeta \bar{\theta} \dot{x})=x\left(\frac{p^{2}+\bar{\theta}^{2}}{p^{2}+2 \zeta \bar{\theta} p+\bar{\theta}^{2}} n\right)
$$

It will therefore be zero when $\bar{\theta} \equiv \theta_{*}$, which is the desired equilibrium. This is true even if in the update law we include only the second right hand term. However, including $x$ gives the algorithm an additional stabilization mechanism that may be unveiled noting that the estimation error can be written as

$$
x(n-2 \zeta \theta \dot{x})=x\left(\ddot{x}+\theta^{2} x\right)
$$

Hence, close to the equilibrium (2.3), where $\theta=\theta_{*}$ and $\ddot{\bar{x}}=-\theta_{*}^{2} \bar{x}$, we have

$$
x(n-2 \zeta \theta \dot{x}) \approx\left(\bar{\theta}^{2}-\theta_{*}^{2}\right) \bar{x}^{2}
$$

The derivations above show that, whenever we are close to the desired orbit and adaptation is slow, the search in parameter space will go in the right direction (i.e., $\left\{\theta>\theta_{*} \Rightarrow \dot{\theta} \leq 0\right\}$, and vice versa). This is the key (local) stabilization mechanism of Regalia's ANF which is rigourously formalized in the next subsection. See also [23] for an alternative discussion of the ANF above using Ljung's ODE analysis.

\subsection{Stability analysis}

As in all adaptive systems, when the adaptation gain $\gamma$ of the ANF update law is small the estimated frequency $\theta$ tends to evolve slowly compared to the filter states $\chi \triangleq[x, \dot{x}]^{T}$. As shown in the fundamental paper [25] this concept of slow adaptation can be made precise by proving that it occurs on an integral manifold of (2.1), (2.2), i.e., a time-varying 1-dimensional surface $M_{\gamma} \subset \mathcal{R}^{3}$ such that

$$
\left(\chi\left(t_{0}\right), \theta\left(t_{0}\right)\right) \in M_{\gamma} \Rightarrow(\chi(t), \theta(t)) \in M_{\gamma}, \quad \forall t \geq t_{0}
$$

The proposition below establishes the existence of a $\gamma$-family of slow manifolds for Regalia's ANF. The simplest member of this family is the "frozen parameter" manifold $M_{0}$, corresponding to $\gamma=0$. We furthermore show that in this manifold the update law is stable.

\footnotetext{
${ }^{3}$ In [2] there is an additional filter gain which can be absorbed into $k$ without affecting the subsequent analysis.

${ }^{4}$ Of course, such a choice is more meaningful if the phase of the sinusoid is zero at $t=t_{0}$. See footnote 1 of the introduction.

${ }^{5}$ Here, and throughout the rest of the paper, we use $(\bar{\cdot})$ to denote equilibria (not necessarily constant).
} 
Proposition 2.1 (Integral manifolds of slow adaptation)

There exists $\gamma_{0}$ such that for each $\gamma \in\left[0, \gamma_{0}\right],(2.1),(2.2)$ has a uniquely defined integral manifold $M_{\gamma}=$ $\{t, \theta, \chi: \chi=f(t, \theta)\}$, which arbitrarily approaches the frozen-parameter manifold $M_{0}$ as $\gamma \rightarrow 0$. The latter can be explicitely characterized and expressed in a time-independent form as a 2-dimensional manifold in $\mathcal{R}^{3}$ given by

$$
M_{0}=\left\{(x, \dot{x}, \theta): x^{2}+\frac{1}{\theta_{*}^{2}} \dot{x}^{2}=\frac{k^{2}}{\left(\theta^{2}-\theta_{*}^{2}\right)^{2}+4 \zeta^{2} \theta^{2} \theta_{*}^{2}}\right\}
$$

Furthermore, on the manifold $M_{\gamma}$ the update law is asymptotically stable in the sense that $\theta \rightarrow \theta_{*}$ as $t \rightarrow \infty$.

\section{Proof}

The proof of existence of the manifolds is a direct application of Theorem 3.1 of [25], and it boils down to verifying the required assumptions. We refer the reader to that paper and to [5] for further details, and to [16] for a similar analysis.

First, we take a state-space realization of (2.1), (2.2) as

$$
\begin{aligned}
\dot{\chi} & =\left[\begin{array}{cc}
0 & 1 \\
-\theta^{2} & -2 \zeta \theta
\end{array}\right] \chi+\left[\begin{array}{l}
0 \\
1
\end{array}\right] n \\
\dot{\theta} & =-\gamma \chi_{1}\left(n-2 \zeta \theta \chi_{2}\right)
\end{aligned}
$$

Let $\chi^{0}(t, \theta)$ be the steady-state response with frozen $\theta$ of $(2.6)$, which can be readily verified to be given by

$$
\chi^{0}(t, \theta)=a(\theta)\left[\begin{array}{c}
\sin \left(\theta_{*} t+\phi(\theta)\right) \\
\theta_{*} \cos \left(\theta_{*} t+\phi(\theta)\right)
\end{array}\right]
$$

where

$$
a(\theta) \triangleq \frac{k}{D(\theta)}, \phi(\theta) \triangleq \arctan \frac{-2 \zeta \theta \theta_{*}}{\theta^{2}-\theta_{*}^{2}}
$$

and

$$
D^{2}(\theta) \triangleq\left(\theta^{2}-\theta_{*}^{2}\right)^{2}+4 \zeta^{2} \theta^{2} \theta_{*}^{2}>0
$$

Notice that $\phi(\theta)$ and $a(\theta)$ are continuous functions of $\theta$.

The proof that the frozen parameter manifold is contained in the set (2.5) follows from direct substitution of (2.7).

Now, introducing the deviation of $\chi$ from $\chi^{0}(t, \theta)$ as a new state variable

$$
y=\chi-\chi^{0}(t, \theta)
$$

we rewrite the ANF equations as

$$
\begin{aligned}
\dot{y} & =\left[\begin{array}{cc}
0 & 1 \\
-\theta^{2} & -2 \zeta \theta
\end{array}\right] y+\gamma \chi_{\theta}^{0}(t, \theta) F(t, \theta, y) \\
\dot{\theta} & =-\gamma\left[\chi_{1}^{0}(t, \theta)+y_{1}\right]\left\{n-2 \zeta \theta\left[\chi_{2}^{0}(t, \theta)+y_{2}\right]\right\} \triangleq-\gamma F(t, \theta, y)
\end{aligned}
$$

where $\chi_{\theta}^{0}(t, \theta)$ is the sensitivity matrix. We can now show that the conditions of theorem 3.1 of [25] hold, namely:

- (Assumption 2.1) The frozen parameter unforced system

$$
\dot{y}=\left[\begin{array}{cc}
0 & 1 \\
-\theta^{2} & -2 \zeta \theta
\end{array}\right] y
$$

is exponentially stable $\forall \theta>0$;

- (Assumption 2.2) $\chi^{0}(t, \theta), \chi_{\theta}^{0}(t, \theta)$ are bounded and the latter is Lipschitzian in $\theta$; 
- (Assumption 3.1) $F(t, \theta, y)$ is continuous, hence bounded in compacts, and Lipschitzian in $\theta$ and $y$.

Invoking theorem 3.1 of [25] this proves the existence of a $\gamma$-family of slow manifolds $M_{\gamma}$ for sufficiently small $\gamma$.

The stability of the update law can be shown as an application of Theorem 4.1 of [25] and Theorem 4.4.3 of [27]. Note that when $y=0$ the update law reduces to

$$
\dot{\theta}=F(t, \theta, 0)=\chi_{1}^{0}(t, \theta)\left[n-2 \zeta \theta \chi_{2}^{0}(t, \theta)\right]=\left(\theta^{2}-\theta_{*}^{2}\right)\left[\chi_{1}^{0}(t, \theta)\right]^{2}
$$

and its averaged form is

$$
\dot{\theta}_{a}=-\gamma\left(\theta_{a}^{2}-\theta_{*}^{2}\right) a\left(\theta_{a}\right) / 2 \triangleq \gamma f_{a}\left(\theta_{a}\right)
$$

where $f_{a}\left(\theta_{a}\right)$ has an isolated zero at $\theta_{a}=\theta_{*}$ and $\left[d f_{a} / d \theta_{a}\right]_{\theta_{a}=\theta_{*}}<0$.

\section{Remark 2.1}

One can further prove the attractivity of the integral manifold $M_{\gamma}$ (Theorem 5.1 of [25]). The existence and stability results established thus far are only local. However, the slow manifold approach was discussed above in some detail because it sets a convenient framework also for the global analysis that we pursue.

\subsection{Simulations: Instability and performance degradation}

In this subsection we present some simulations ${ }^{6}$ that illustrate the result of proposition 2.1, and reveal two potential problems for Regalia's ANF that appear for small and large frequencies, respectively. This two phenomena motivate our first modification, scaling, which is presented in section 3 .

The existence of the slow adaptation manifold is depicted in Figs. 1, 2 and 3, where the latter clearly shows the vertex-up cone form of the frozen-parameter manifold (2.5). The fast convergence of the trajectory towards the slow manifold, and the subsequent drift along it towards the desired orbit $(t>5)$, is best appreciated in the plot of $\theta \times t$ of Fig. 2. The initial conditions (IC) in this simulation are $\left[x_{1}, x_{2}, x_{3}\right]=$ $[1,1,10]$, and the remaining parameters $\gamma=0.1, \zeta=0.1, \theta_{*}=5, k=10$. Note the periodic orbit at $\theta=\theta_{*}$ with amplitude $\frac{k}{2 \zeta \theta_{*}^{2}}=2$ for $x_{1}$.

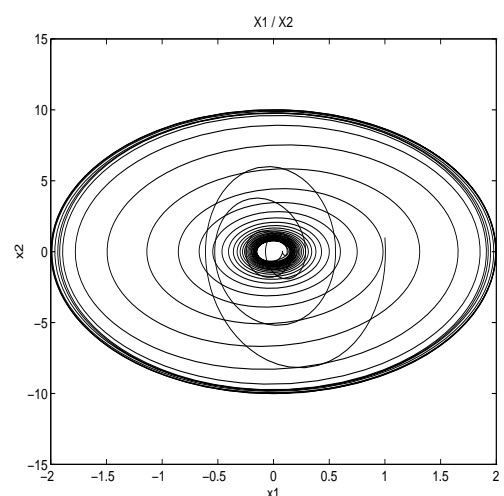

Figure 1: $\left(x_{1}, x_{2}\right)$ plane

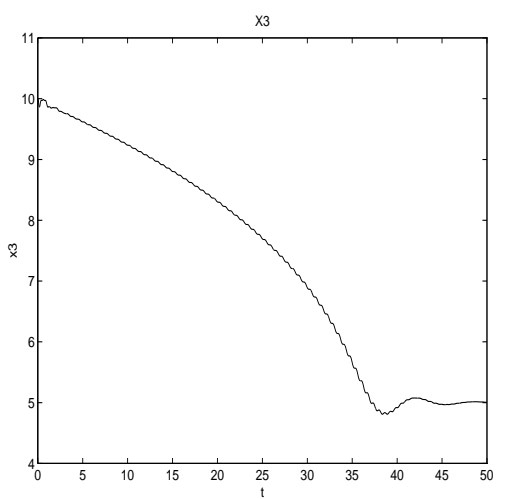

Figure 2: $\theta(t)\left(\theta_{*}=5\right)$

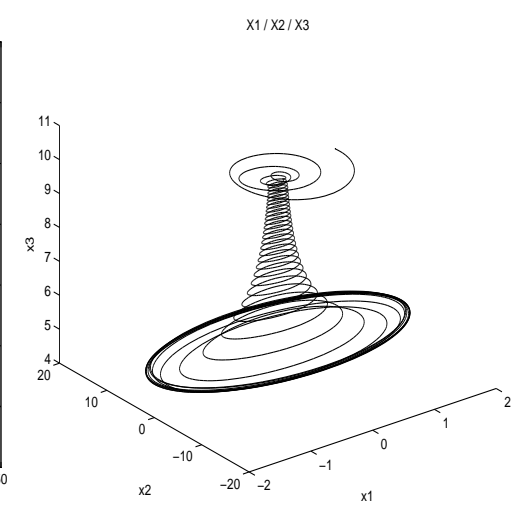

Figure 3: 3D state-space plot

Since the amplitude of the periodic orbit (2.3) grows unboundedly with $\theta_{*} \rightarrow 0$ and decreases to zero as $\theta_{*} \rightarrow \infty$, two problems may arise. If $\theta_{*}$ is large, the amplitude may be so small that the convergence becomes very slow. This is shown in Figs. 4, 5 and 6 , where the ICs are taken as $[1,1,10], \gamma=0.5, \zeta=0.4$, $\theta_{*}=100$ and $k=10$. We have let the simulation run up to 10000 to verify that parameter convergence was not yet achieved.

\footnotetext{
${ }^{6}$ In all simulations we denote $\left(x_{1}, x_{2}, x_{3}\right)=(x, \dot{x}, \theta)$.
} 


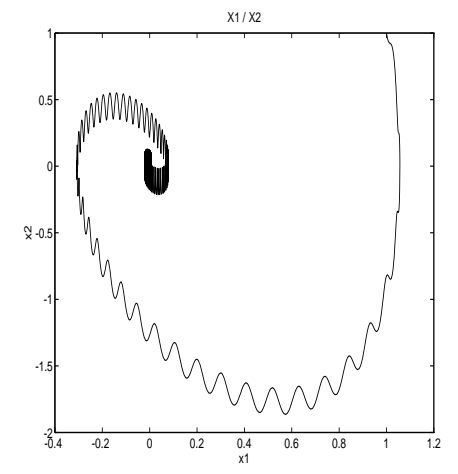

Figure 4: $\left(x_{1}, x_{2}\right)$ plane (large $\left.\theta_{*}=100\right)$

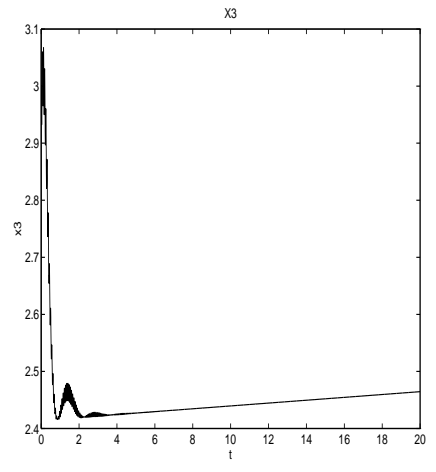

Figure 5: $\theta(t)$

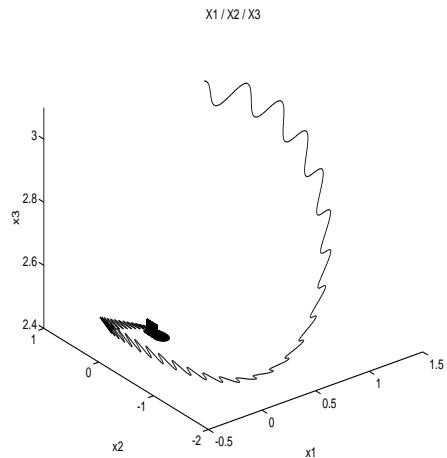

Figure 6: 3D state-space plot

On the other hand, with small $\theta_{*}$, large oscillations may provoke unpredictable behavior and trigger instability. This can be seen in Figs. $7,8,9$ where we have taken $\theta_{*}=0.1$, keeping the remaining conditions and parameters as in the previous case.

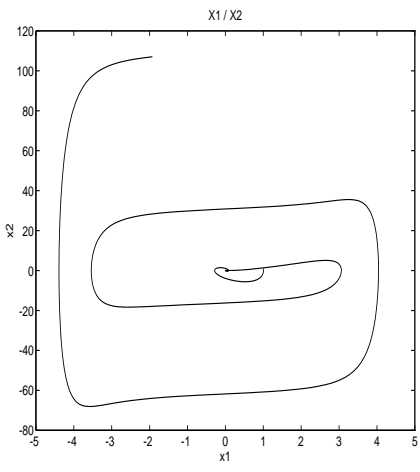

Figure 7: $\left(x_{1}, x_{2}\right)$ plane (small $\left.\theta_{*}=0.1\right)$

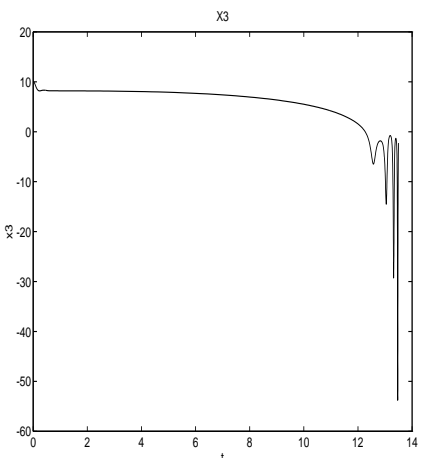

Figure 8: $\theta(t)$

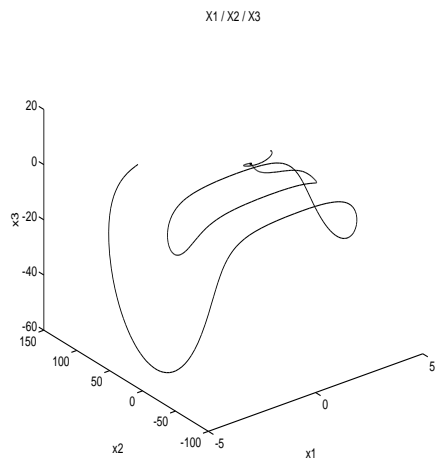

Figure 9: 3D state-space plot

Regalia's ANF is only locally stable, it may go unstable for large ICs. For instance, it can be shown by simulation that for $\gamma=0.7, \zeta=0.3, \theta_{*}=5, k=1$ and IC $[1,1,10]$, the system has stable behaviour and convergence to the correct frequency is observed. However, it is shown in [5] that with IC [10,1,10], the trajectory blows away.

\section{Remark 2.2}

It is clear from the simulations (e.g., Fig. 8 above or Fig. 2 of [2]) that nothing prevents the estimate $\theta$ from taking negative values during the transient. As shown above this triggers instability. Even though, a simple projection could be added to avoid this to happen, there's no guarantee that an acummulation point of the algorithm occurs close to zero whence the projection will be turning on and off indefinitely. This is a very delicate well known problem in adaptive systems that we want to avoid in our global (fully deterministic) analysis.

\section{Scaled adaptive notch filter}

Motivated by the discussions above, we propose in this section our first modification to Regalia's ANF:

- Scaling of the forcing signal to obtain a "unitary gain" in steady-state and avoid the undesirable crossing through zero of the estimated frequency (remark 2.2). 
After presenting the modified scheme, which we called scaled-ANF, we prove the positivity of the estimate and verify, via simulation, how the instability problems mentioned above are avoided.

\subsection{Proposed modification and positivity of the estimate}

We propose to modify Regalia's ANF (2.1), (2.2) by scaling the forcing term $n$ with a factor $\theta^{2}$ to get

$$
\begin{aligned}
\ddot{x}+2 \zeta \theta \dot{x}+\theta^{2} x & =\theta^{2} n \\
\dot{\theta} & =-\gamma\left(\theta^{2} n-2 \zeta \theta \dot{x}\right) x
\end{aligned}
$$

Using the same reasoning as in subsection 2.1 it is easy to see that, under ideal conditions, the amplitude of $x$ will converge to $k / 2 \zeta$, instead of $k / 2 \zeta \theta_{*}^{2}$ as in Regalia's algorithm, thus avoiding the instability and the slow convergence that appear for low and high frequencies, respectively.

Another nice feature of the new algorithm is that the frequency search is restricted to non-negative values. That is, along the solutions of (3.1), (3.2) the following implication holds

$$
\theta\left(t_{0}\right)>0 \Rightarrow \theta(t)>0, t \in\left[t_{0}, \infty\right)
$$

This is, of course, a direct consequence of the invariance of the set $\{\theta \equiv 0\}$.

Besides its obvious practical interest this property will be important in the next section where, to decouple the filter and the estimator dynamics, we will introduce a time scale change that involves a division by $\theta$.

\section{Remark 3.1}

Proceeding as done in proposition 2.1 it is possible to show the existence of the slow manifolds for the scaled ANF. In this case the steady-state response with frozen $\theta$ is given by (2.7) but with $a(\theta)=k \theta^{2} / D(\theta)$ instead of (2.8). Hence the frozen parameter manifold looks like

$$
M_{0}=\left\{(x, \dot{x}, \theta): x^{2}+\frac{1}{\theta_{*}^{2}} \dot{x}^{2}=\frac{k^{2} \theta^{2}}{\left(\theta^{2}-\theta_{*}^{2}\right)^{2}+4 \zeta^{2} \theta^{2} \theta_{*}^{2}}\right\}
$$

It is interesting to note that the cone is now inverted. It is easy to see, also, that the fundamental relationship (2.10) still holds true. We will come back to this in section 5 where we will analyse the scaled and normalized ANF.

\subsection{Simulations}

The improved performance of the new scaled ANF, for large $\theta_{*}$, can be seen in Figs.10-12. We used the same data of Figs. 4-6, for which Regalia's ANF exhibited very poor convergence properties. We can see that the oscillations reasonably soon achieve large enough values, thus resulting in much faster transient response.

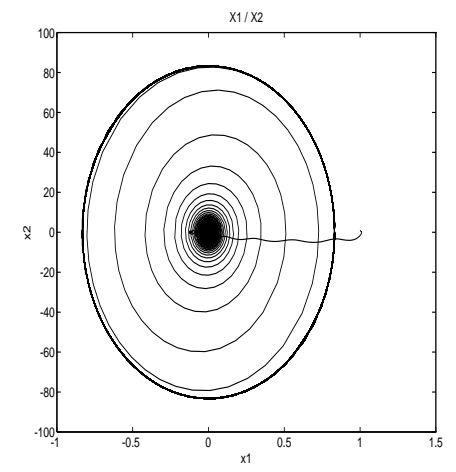

Figure 10: $\left(x_{1}, x_{2}\right)$ (large $\theta_{*}=$ 100)

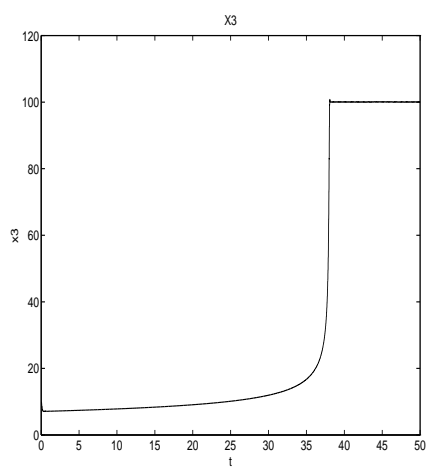

Figure 11: $\theta(t)$

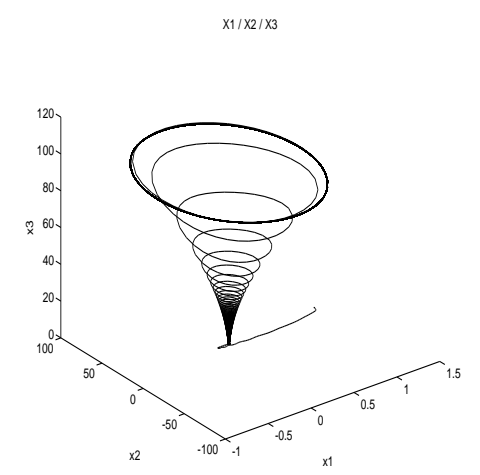

Figure 12: 3D state-space plot 
For small $\theta_{*}$, the scaled system also exhibits good behaviour mainly because the oscillation amplitudes are not so large as in the original ANF. Thus, a fast and nice convergence is observed. See Figs. 13-15, which should be compared with Figs. 7-9. Again, the same data are used to produce both simulations.

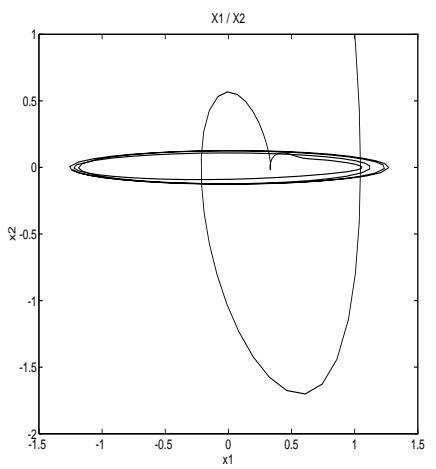

Figure 13: $\left(x_{1}, x_{2}\right)$ (small $\theta_{*}=$ $0.1)$

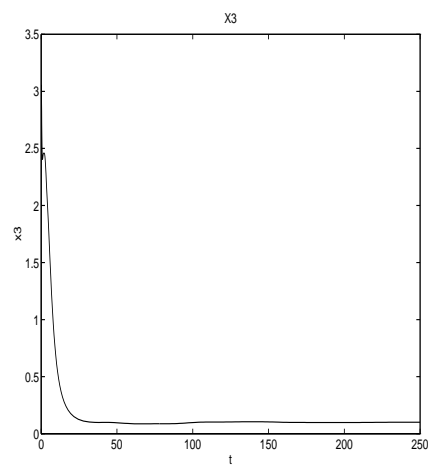

Figure 14: $\theta(t)$

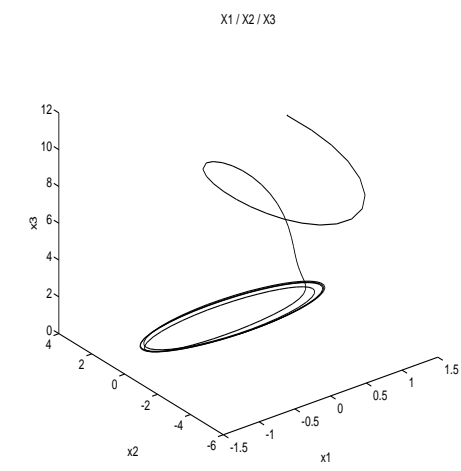

Figure 15: 3D state-space plot

Even though scaling has removed two important drawbacks of Regalia's ANF it is verified in [5] that the new estimator is still only locally stable.

\section{Time scale change and normalization}

To guide ourselves in the modification to the ANF required to achieve globality we propose in this section a nonlinear time scale change that decouples the dynamics of the estimator and the filter. More precisely, in this time scale we can represent the ANF as a feedback interconnection of a stable LTI system with a "nonlinear gain", which can be bounded with the introduction of a suitable normalization factor in the estimator. In this way we can prove that, for the normalized and scaled ANF, the filter signals are globally bounded. The proof of asymptotic convergence of the estimate, being more technically involved, is left to section 5. Even though it may be argued that normalization is a standard modification to achieve globality, it will become clear below that the required form of the normalization is far from obvious, and becomes only clear in the new time scale.

\subsection{Notation and preliminaries}

Time scaling, whose importance in the study of dynamical systems is recognized already in Lyapunov's original work [15], plays a fundamental role in the theory developed here and is used in the remaining of the paper. Some simplifying notations will be introduced as follows. Consider a system given by

$$
\frac{d x(t)}{d t}=f(x(t), t), x\left(t_{0}\right)=x_{0} \in \mathcal{R}^{n}
$$

Then, define the time-scale transformation

$$
\frac{d \tau}{d t}=g(x(t), t) ; \quad \tau\left(t_{0}\right)=t_{0}
$$

where, $\tau$ is the new time variable, $x(t)$ is a solution of (4.1), and $g(x(t), t)>0, \forall t \in I_{M}=\left[t_{0}, t_{M}\right)$, with $t_{0}$ being an initial time and $t_{M}$ the upper limit of the maximal interval of definition of $x(t)$ as a function of $t$. This defines (under the usual assumptions of existence and uniqueness of solutions) a homeomorphism $\mathcal{R} \rightarrow \mathcal{R}$ relating the time variables as

$$
\tau=\tau(t) ; t=t(\tau)
$$

In terms of the new time variable, the original system is transformed into

$$
\frac{d x_{\tau}(\tau)}{d \tau}=f g^{-1}\left(x_{\tau}(\tau), t\right)
$$




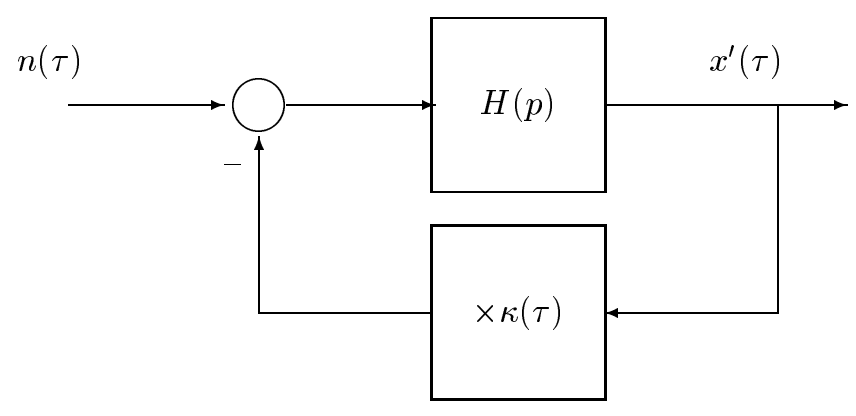

Figure 16:

The notational simplifications we adopt in this paper are: $x_{\tau}(\tau)$ is simply replaced by $x(\tau)$. Also, we use $(.) \triangleq \frac{d(.)}{d t}$ and $(.)^{\prime} \triangleq \frac{d(.)}{d \tau}$. When clear from the context we will use the symbol $p$ to denote differentiation with respect to either $t$ or $\tau$. To clearly distinguish between the time scales we will explicitly write the argument $(\tau)$ whenever the signal is expressed in that time scale.

Note that, if $x(t)$ is a solution of the original system (4.1), then $x_{\tau}(\tau)=x(t(\tau))$ is a solution of the transformed system above. Since we will study the stability of (4.1) using its $\tau$-scale representation

$$
x^{\prime}(\tau)=f g^{-1}(x(\tau), t(\tau))
$$

we must prove that the new time variable $\tau=\tau(t)$ is defined $\forall t$, nondecreasing and satisfies

$$
\tau \rightarrow \infty \Leftrightarrow t \rightarrow \infty
$$

Establishing this fact is a difficult technical hurdle of our analysis that will, unfortunately, translate into some additional complexity in the algorithm.

\subsection{Estimator and filter decoupling}

\section{Proposition 4.1}

Let us define a (state-dependent) time scale change as

$$
\frac{d \tau(t)}{d t}=\theta(t) ; \quad \tau\left(t_{0}\right)=\tau_{0}
$$

with $\theta\left(t_{0}\right)>0$. Then, in the $\tau$ time scale and $\forall \tau \geq \tau_{0}$, the dynamics of the scaled ANF (3.1), (3.2) are described by the following 4 -dimensional autonomous system

$$
\begin{aligned}
x^{\prime \prime}(\tau)+2 \zeta x^{\prime}(\tau)+[1 & \left.+2 \gamma \zeta\left(x^{\prime}(\tau)\right)^{2}\right] x(\tau)=\left[1+\gamma x(\tau) x^{\prime}(\tau)\right] n(t(\tau)) \\
\theta^{\prime}(\tau) & =\gamma\left[2 \zeta x^{\prime}(\tau)-n(t(\tau))\right] x(\tau) \theta(\tau) \\
t^{\prime}(\tau) & =\frac{1}{\theta(\tau)}
\end{aligned}
$$

with initial conditions ${ }^{7}\left(x\left(\tau_{0}\right), x^{\prime}\left(\tau_{0}\right), \theta\left(\tau_{0}\right), t\left(\tau_{0}\right)\right)=\left(x\left(t_{0}\right), \frac{\dot{x}\left(t_{0}\right)}{\theta\left(t_{0}\right)}, \theta\left(t_{0}\right), t_{0}\right)$.

In particular, the filter equations (4.4) can be represented as the feedback interconnection of Fig. 16 where

and

$$
H(p) \triangleq \frac{p}{p^{2}+2 \zeta p+1}
$$

$$
\kappa(\tau) \triangleq \gamma x(\tau)\left[2 \zeta x^{\prime}(\tau)-n(t(\tau))\right]
$$

\footnotetext{
${ }^{7}$ Notice that, even though $x$ and $x(\tau)$ denote the same function in different time scales, this is not so for $\dot{x}$ and $x^{\prime}(\tau)$, hence the difference in initial conditions.
} 
Proof

The proof is a straightforward application of the chain rule

$$
(\cdot)^{\prime}(\tau)=(\cdot) \frac{1}{\theta(\tau)}
$$

and the incorporation of $t(\tau)$ as a state variable. Application of the time scale change to (3.1) yields

$$
x^{\prime \prime}(\tau)+\left[2 \zeta+\frac{\theta^{\prime}(\tau)}{\theta(\tau)}\right] x^{\prime}(\tau)+x(\tau)=n(t(\tau))
$$

while (3.2) becomes (4.5). The proof is completed replacing (4.5) in the expression above and rearranging the terms in (4.4) to obtain the feedback system of Fig. 16.

\section{Remark 4.1}

It is useful to rewrite (4.4) as

$$
x^{\prime \prime}(\tau)+x(\tau)+\left[1+\gamma x^{\prime}(\tau) x(\tau)\right]\left[2 \zeta x^{\prime}(\tau)-n(t(\tau))\right]=0
$$

From simple inspection of the equation above and (4.5) we see that, in the $\tau$ time scale, the unique solution which ensures the estimator equation is in steady-state is

$$
\left[\begin{array}{c}
\bar{x}(\tau) \\
\bar{x}^{\prime}(\tau) \\
\bar{\theta} \\
\bar{t}(\tau)
\end{array}\right]=\left[\begin{array}{c}
\frac{-k}{2 \zeta} \cos (\tau) \\
\frac{k}{2 \zeta} \sin (\tau) \\
\theta_{*} \\
\frac{1}{\theta_{*}} \tau
\end{array}\right]
$$

\subsection{Normalization and global boundedness}

From Fig. 16, and provided the time scaling is well defined, we see that if the gain $\kappa(\tau)$ is bounded which can be achieved with a suitable selection of $\gamma$ - we can use a simple $\mathcal{L}_{\infty}$ small-gain argument to prove boundedness of the feedback loop. This reasoning motivates the introduction in this subsection of our second modification: normalization of the update law. This allows us to achieve global boundedness of the filter signals. The proof of boundedness and convergence of $\theta$ is, unfortunately, more technical and requires the assumption of slow adaptation. Therefore, it is postponed to section 5 .

\section{Proposition 4.2}

Consider the scaled normalized ANF

$$
\begin{gathered}
\ddot{x}+2 \zeta \theta \dot{x}+\theta^{2} x=\theta^{2} n \\
\dot{\theta}=\gamma(2 \zeta \dot{x}-\theta n) x \theta \\
\gamma=\frac{\epsilon}{\left\{1+N\left[x^{2}+\left(\frac{\dot{x}}{\theta}\right)^{2}\right]\right\}\left\{1+\mu|\theta|^{\alpha}\right\}}
\end{gathered}
$$

where, $\epsilon, N, \mu>0$ and $\alpha \geq 1$. We propose the following state realization ${ }^{8}$ for (4.10)-(4.12)

$$
\begin{aligned}
\dot{z}_{1} & =\theta z_{2} \\
\dot{z}_{2} & =\theta\left[-z_{1}-2 \zeta z_{2}+n-\gamma z_{1} z_{2}\left(2 \zeta z_{2}-n\right)\right] \\
\dot{\theta} & =\gamma\left(2 \zeta z_{2}-n\right) z_{1} \theta^{2} \\
\gamma & =\frac{\epsilon}{\left(1+N \rho_{z}^{2}\right)\left(1+\mu|\theta|^{\alpha}\right)}
\end{aligned}
$$

where $z_{1}=x, z_{2}=x^{\prime}$ and $\rho_{z}^{2} \triangleq z_{1}^{2}+z_{2}^{2}$.

\footnotetext{
${ }^{8}$ Notice that, in contrast to $(2.6)$, this is not a canonical state-space realization since $x_{2}$ is not the derivative of $x_{1}$. This particular form will be needed for the subsequent analysis.
} 
Assume known an upperbound $k_{M}$ of the sinusoidal noise amplitude, i.e., $k \leq k_{M}$, fix $\zeta>0$, and choose $\epsilon, N$ such that

$$
\epsilon<\frac{2 N}{k_{M} N^{1 / 2}+4 \zeta}\|h\|_{1}
$$

where $h$ is the impulse response of the transfer function $(4.7)$ and $\|\cdot\|_{1} \triangleq \int_{0}^{\infty}|\cdot| d t$ is its $\mathcal{L}_{1}$ norm. Under these conditions,

i) The time scale change (4.3) is globally defined, i.e., (4.2) holds;

ii) $z_{1}$ and $z_{2}$ are globally bounded.

\section{Proof}

Positivity of the estimate, stated in fact 3.1 , shows that $\tau$ is a strictly increasing function, thus to prove i), we need only to rule out the possibilities of $\tau$ going to infinity in finite time or that $\tau$ converges to a finite constant as $t \rightarrow \infty$. These scenarios correspond to the cases when $\theta$ escapes to infinity in finite time and $\tau-\tau_{0}=\int_{t_{0}}^{t} \theta(s) d s \rightarrow c<\infty$ as $t \rightarrow \infty$, respectively. To prove that $\theta$ does not escape to infinity in finite time, let us rewrite (4.11) as

$$
\dot{\theta}=\kappa_{1} \theta
$$

where we have defined

$$
\kappa_{1} \triangleq \gamma x(2 \zeta \dot{x}-\theta n)
$$

Some simple calculations with (4.19) yield

$$
\left|\kappa_{1}\right| \leq \kappa_{1_{M}} \triangleq \frac{\epsilon}{2 N}\left(k_{M} N^{1 / 2}+4 \zeta\right) \mu^{-1 / \alpha} c_{\theta}
$$

where $\mu^{-1 / \alpha} c_{\theta}(\alpha) \triangleq \sup _{\theta} \frac{|\theta|}{\left(1+\mu|\theta|^{\alpha}\right)}=\mu^{-1 / \alpha}(1-1 / \alpha)(\alpha-1)^{-1 / \alpha}<\infty$. Integrating (4.18) and using the bound above we get

$$
\theta \leq \mathrm{e}^{\epsilon \kappa_{1} M^{t}} \theta(0)
$$

which proves that $\theta$ does not escape to infinity in finite time.

We will now prove that ${ }^{9} \lim _{t \rightarrow \infty} \tau=\infty$. We will proceed by contradiction, thus assume that $\lim _{t \rightarrow \infty} \tau=$ $\tau_{M}<\infty$. Then, notice that the developments carried out for the time scaling in proposition 4.1 remain valid for time-varying $\gamma$. Henceforth, (4.10), (4.11) become (4.4)-(4.6), with

$$
\gamma(\tau)=\frac{\epsilon}{\left[1+N \rho_{z}^{2}(\tau)\right]\left(1+\mu|\theta(\tau)|^{\alpha}\right)}
$$

In particular, in the $\tau$ time scale, the estimator (4.11) is described by $\theta^{\prime}(\tau)=\kappa(\tau) \theta(\tau)$. Some simple calculations with (4.8) show that $\kappa(\tau)$ satisfies the bound

$$
|\kappa(\tau)|<\frac{\epsilon}{2 N}\left(k_{M} N^{1 / 2}+4 \zeta\right)
$$

We draw two important conclusions here. First, from (4.20) we see that $\theta^{\prime}$ is linearly bounded and thus $\theta(\tau)$ cannot decrease to zero faster than exponentially and thus $\theta=0$ cannot be reached in finite time. This implies that the open set $\theta>0$ is invariant. Note that system (4.4)-(4.6) is singular for $\theta=0$, however, for the open set $\theta>0$ the system is locally continuously differentiable, which guarantees (local) existence and uniqueness of solutions within the invariant set. Second, again as $\theta(t), \theta(\tau)$ can grow at most exponentially. The latter implies that $\theta(\tau)$ and $\theta^{\prime}(\tau)$, are bounded for $\tau \in\left[0, \tau_{M}\right)$. This in turn implies that $\theta(t)$ and $\dot{\theta}(t)$ are bounded $\forall t \geq 0$. Hence, $\theta(t)$ is uniformly continuous. Integrating (4.3) from 0 to $\infty$ and invoking Barbalat's lemma [27], one concludes that $\theta(\tau) \rightarrow 0$ as $\tau \rightarrow \tau_{M}$.

This situation cannot happen because, as already pointed out, $\theta(\tau)$ can decrease to zero at most exponentially, and consequently, $\theta(\tau)$ remains bounded away from zero (or is identically equal to zero) for any

\footnotetext{
${ }^{9}$ Recall that $\tau(t)$, being strictly increasing in $t$, either is unbounded or tends to a finite constant.
} 
finite interval $\left[0, \tau_{M}\right)$. The contradiction is therefore established thus proving the global definition of the time scale change.

To prove the global boundedness result ii), (in the $\tau$ time scale), we refer to the feedback system of Fig. 16. Recalling that the $\mathcal{L}_{\infty}$ gain of an LTI operator is the $\mathcal{L}_{1}$ norm of its impulse response [6], and combining (4.17), (4.20) we establish boundedness of $x^{\prime}(\tau)$ as a straightforward application of the $\mathcal{L}_{\infty}$ smallgain theorem. Also, notice that $z_{1}(\tau)=x(\tau)$ is the output of an asymptotically stable linear time invariant system with bounded input $n(\tau)-\kappa(\tau) x^{\prime}(\tau)$, hence is also bounded. The proof is completed observing that $z_{2}=\frac{\dot{x}}{\theta}=x^{\prime}(\tau)$, whose boundedness we just established.

\section{Remark 4.2}

Normalization was originally motivated by our concern to bound the gain $\kappa(\tau)$ in Fig. 16 and prove global boundedness. It is clear from (4.8) that to achieve this end it suffices to include the first two terms in brackets in (4.12). The remaining term is needed, as shown in the proof, to avoid finite escape time phenomena. Observe, however, that $\mu$ may be taken arbitrarily small.

\section{Global convergence of the estimate}

In this section we prove the main result of the paper:

\section{Proposition 5.1}

There exists $\epsilon_{*}>0$ satisfying the bound ${ }^{10}$

$$
\epsilon_{*}<\frac{2 N}{k_{M} N^{1 / 2}+4 \zeta}\|h\|_{1}
$$

such that for all $\epsilon \leq \epsilon_{*}$ all signals of the new ANF (4.13)-(4.16) are globally bounded for arbitrary initial conditions. Furthermore, the estimated frequency is asymptotically correct, that is $\lim _{t \rightarrow \infty} \theta=\theta_{*}$.

To carry out the proof we will proceed along the following steps (for each one of which a subsection is devoted below):

1. As done in section 2.2 (slow manifold approach), we evaluate the quasi-static solution of the new ANF, (i.e., its steady-state response with frozen $\theta$ ), and define as error signals the deviation of the actual ANF signals with respect to this quasi-static solution. The error system will be of the following form

$$
\begin{aligned}
& y^{\prime}(\tau)=A y(\tau)+\epsilon h(\tau, y, \theta) \\
& \theta^{\prime}(\tau)=\epsilon f(\tau, y, \theta)
\end{aligned}
$$

which is in the standard separated time scales form of averaging theory [27].

2. We prove that the error subsystem (5.1) may be regarded as an exponentially stable LTI system perturbed by small (order $\epsilon$ ) parametric and external disturbances. Hence, under sufficiently slow adaptation, the actual signals $z_{1}(\tau)$ and $z_{2}(\tau)$ of the scaled and normalized ANF get arbitrarily close to the quasi-static solutions. This means that, after some finite time, $y(\tau)$ will be constrained to be small.

3. We decompose the estimator equation into a nominal system and a perturbation as

$$
f(\tau, y, \theta)=f_{0}(\tau, y, \theta)+f_{1}(\tau, y, \theta)
$$

with $\theta_{*}$ an asymptotically stable equilibrium point for the nominal system and the perturbing term is order $\epsilon\|y(\tau)\|$. Henceforth, once $y(\tau)$ is small, one can prove that $\theta(\tau)$ will have to reach an $\epsilon$ neighbourhood of the ideal value $\theta_{*} .^{11}$

\footnotetext{
${ }^{10}$ See proposition 4.2 for the definition of the right hand terms.

${ }^{11}$ Unfortunately, the lack of exponential stability of the nominal system hampers us from invoking standard perturbation results to establish this result, hence an additional technical lemma is required.
} 
4. We show that the periodic orbit is locally exponentially stable with a domain of stability $\mathcal{D}$, which is valid for all sufficiently small $\epsilon$ (via standard averaging theory). This implies that the complete error vector will eventually enter $\mathcal{D}$, and then exponential convergence of the error vector to zero follows.

\subsection{Error equations}

As pointed out in remark 3.1 the quasi-static solution of the new ANF differs from the one of Regalia's ANF only on a factor $\theta^{2}$. Hence, for the state space realization (4.13)-(4.15) we get

$$
\left[\begin{array}{c}
z_{1}^{0}(\theta, t) \\
z_{2}^{0}(\theta, t)
\end{array}\right]=a(\theta) \theta^{2}\left[\begin{array}{c}
\sin \left(\theta_{*} t+\phi(\theta)\right) \\
\frac{\theta_{*}}{\theta} \cos \left(\theta_{*} t+\phi(\theta)\right)
\end{array}\right]
$$

where $a(\theta), \phi(\theta)$ are given by (2.8). Notice that, in this state realization ${ }^{12}$

$$
z_{2}^{0}=\frac{1}{\theta} \frac{\partial z_{1}^{0}}{\partial t}
$$

which explains the presence of $1 / \theta$ in the second component.

We underscore at this point that the local stabilization mechanism discussed in section 2.2, which ensures the stable behaviour of the estimator in the frozen parameter manifold, is also present in the new ANF. This is clear from the identity

$$
\left(2 \zeta z_{2}^{0}-n\right) z_{1}^{0}=-\left[1-\left(\frac{\theta_{*}}{\theta}\right)^{2}\right]\left(z_{1}^{0}\right)^{2}
$$

We denote again the error vector $y=\left[\begin{array}{ll}y_{1} & y_{2}\end{array}\right]^{T} \triangleq\left[\begin{array}{lll}z_{1}-z_{1}^{0} & z_{2}-z_{2}^{0}\end{array}\right]^{T}$. Our motivation to study the behaviour of $y$ becomes clear if we rewrite the estimator equation (4.11) in a form that exhibits explicitely the stabilization mechanism captured by (5.5). Towards this end, we decompose it as

$$
\begin{aligned}
\dot{\theta} & =\gamma\left(2 \zeta z_{2}-n\right) z_{1} \theta^{2} \\
& =\gamma\left[\left(2 \zeta z_{2}-n\right) y_{1}+2 \zeta z_{1}^{0} y_{2}+\left(2 \zeta z_{2}^{0}-n\right) z_{1}^{0}\right] \theta^{2} \\
& =\epsilon\left(g_{0}+g^{T} y\right) \theta^{2}
\end{aligned}
$$

where we have defined

$$
\begin{aligned}
\epsilon g_{0} & \triangleq-\gamma\left(z_{1}^{0}\right)^{2}\left[1-\left(\frac{\theta_{*}}{\theta}\right)^{2}\right] \\
\epsilon g & \triangleq \epsilon\left[\begin{array}{c}
g_{1} \\
g_{2}
\end{array}\right]=\gamma\left[\begin{array}{c}
2 \zeta z_{2}-n \\
2 \zeta z_{1}^{0}
\end{array}\right]
\end{aligned}
$$

In appendix A we show that $g$ is bounded. Consequently, if we can prove that the ANF signals get close to the quasi-static solutions, i.e., that $\|y\|$ becomes small, then the estimator dynamics will be dominated by the term $\dot{\theta}=\epsilon g_{0} \theta^{2}$. In the proof of proposition 2.1 we established that this equation is asymptotically stable.

From (4.13)-(4.15), and using the properties of the quasi-static solution we obtain the error equations

$$
\begin{aligned}
& \dot{y}_{1}=\theta y_{2}-\frac{\partial z_{1}^{0}}{\partial \theta} \dot{\theta} \\
& \dot{y}_{2}=\theta\left(-y_{1}-2 \zeta y_{2}\right)-\gamma \theta z_{1} z_{2}\left(2 \zeta z_{2}-n\right)-\frac{\partial z_{2}^{0}}{\partial \theta} \dot{\theta}
\end{aligned}
$$

We can transform this system using the time scale $\tau,(4.8)$ and the definition of $y_{2}(\tau)$ to obtain

$$
\begin{aligned}
& y_{1}^{\prime}(\tau)=y_{2}(\tau)-\frac{\partial z_{1}^{0}(\tau)}{\partial \theta} \theta^{\prime}(\tau) \\
& y_{2}^{\prime}(\tau)=-y_{1}(\tau)-2 \zeta y_{2}(\tau)-\kappa(\tau)\left[y_{2}(\tau)+z_{2}^{0}(\tau)\right]-\frac{\partial z_{2}^{0}(\tau)}{\partial \theta} \theta^{\prime}(\tau)
\end{aligned}
$$

\footnotetext{
${ }^{12}$ For the sake of brevity in the sequel we will omit the arguments $(\theta, t)$ of the quasi-static solution.
} 
Finally, we express the error equations in the compact form

$$
y^{\prime}(\tau)=\left[A_{0}+\epsilon A_{1}(\tau)\right] y(\tau)+\epsilon b_{1}(\tau) g_{0}(\tau)
$$

where

$$
A_{0} \triangleq\left[\begin{array}{cc}
0 & 1 \\
-1 & -2 \zeta
\end{array}\right], A_{1}(\tau) \triangleq\left[\begin{array}{cc}
0 & 0 \\
0 & -\frac{1}{\epsilon} \kappa(\tau)
\end{array}\right]+b_{1}(\tau) g^{T}(\tau)
$$

with

$$
b_{1}(\tau) \triangleq\left[\begin{array}{c}
-\theta(\tau) \frac{\partial z_{1}^{0}(\tau)}{\partial \theta} \\
-z_{2}^{0}(\tau)-\theta(\tau) \frac{\partial z_{2}^{0}(\tau)}{\partial \theta}
\end{array}\right]
$$

\subsection{Stability of the error equations}

The lemma below establishes the desired properties for the error signals.

\section{Lemma 5.1}

There exists a finite time $\tau_{r} \geq 0$, such that the error signals of the scaled and normalized ANF (4.13)-(4.16) satisfy the bound

$$
\|y(\tau)\| \leq \epsilon \quad ; \quad \forall \tau \geq \tau_{r}
$$

for arbitrary initial conditions provided $\epsilon$ is sufficiently small. Moreover, there exists positive constants $^{13} c$ and $\alpha$ such that

$$
\|y(\tau)\| \leq \epsilon\left[e^{-\alpha \tau} *\left|g_{0}(\tau)\right|+c e^{-\alpha \tau}\right]
$$

where $*$ denotes the convolution operator.

\section{Proof}

In the appendices A and B we show that, similarly to $\kappa(\tau), g_{0}(\tau)$ and the vector $b_{1}(\tau)$ are bounded. Therefore, the subsystem (5.8) can be regarded as an exponentially stable linear time-invariant system perturbed by small parametric and external disturbances. The proof is then standard and based on the quadratic Lyapunov function $V(\tau)=y^{T}(\tau) P y(\tau)$, with

$$
A_{0}^{T} P+P A_{0}^{T}=-Q ; \quad P, Q>0
$$

The derivative of this Lyapunov function gives

$$
\begin{aligned}
V^{\prime}(\tau) & =-y^{T}(\tau) Q y(\tau)+y^{T}(\tau)\left(A_{1}^{T} P+P A_{1}\right) y(\tau)+\epsilon b_{1}^{T}(\tau) P y(\tau) g_{0}(\tau) \\
& \leq-\left(\lambda_{0}-\epsilon\right)\|y(\tau)\|^{2}+\epsilon\|y(\tau)\|\left|g_{0}(\tau)\right|
\end{aligned}
$$

from this inequality and the boundedness of $g_{0}(\tau)$ (5.9) follows immediately. To establish (5.10) we invoke the comparison method (see, e.g., section 5.4 of [13]).

\subsection{Behaviour of the estimate}

Once we have shown that the filter signals $z_{1}, z_{2}$ get arbitrarily close to the quasi-static solutions, remaining close after some finite time $\tau_{r}$ (or $t_{r}$ ), we will next analyze the behavior of the estimated frequency $\theta$. We will show that, no matter its initial condition at time $\tau_{r}$, the estimate will necessarily approach arbitrarily close the correct value $\theta_{*}$ at some finite future time.

\footnotetext{
${ }^{13}$ To avoid the proliferation of constants we will use $c$ as a generic notation for a positive constant and eventually let $\epsilon$ absorb a constant factor $c$, i.e., use simply $\epsilon$ instead of $c \epsilon$.
} 


\section{Lemma 5.2}

The estimated frequency $\theta$ of the scaled and normalized ANF (4.13)-(4.16) is bounded for arbitrary initial conditions provided $\epsilon$ is sufficiently small. Further, for any arbitrarily small positive number $\eta, \exists \tau_{c} \geq \tau_{r}$ such that $\left|\theta\left(\tau_{c}\right)-\theta_{*}\right| \leq \eta .^{14}$

\section{Proof}

We know, from lemma 5.1, that $\exists \tau_{r}$ such that $\|y(\tau)\| \leq \epsilon, \forall \tau \geq \tau_{r}$. First, assume that $\theta\left(\tau_{r}\right)>\theta_{*}$. We will show that $\theta(\tau)$ cannot remain bounded away from $\theta_{*}$, for all increasing $\tau$. Assume that this is not the case, i.e., $\exists \beta>0$ such that, $\theta(\tau)>\beta+\theta_{*}, \forall \tau>\tau_{r}$. In this case, $g_{0}(\tau) \leq 0, \forall \tau>\tau_{r}$.

Now, the estimator equation in the $\tau$ time scale reads like

$$
\theta^{\prime}(\tau)=\epsilon\left[g_{0}(\tau)+g^{T}(\tau) y(\tau)\right] \theta(\tau)
$$

We can integrate this equation, use the sign property of $g_{0}(\tau)$ above and invoke the bound (5.10) to get

$$
\begin{aligned}
\ln \left[\frac{\theta(\tau)}{\theta\left(\tau_{r}\right)}\right] & \leq \int_{\tau_{r}}^{\tau} \epsilon\left[g_{0}-\epsilon\left(e^{-\alpha s} * g_{0}\right)\right] d s+c \epsilon^{2} \\
& \triangleq h_{\epsilon} * g_{0}+c \epsilon^{2}
\end{aligned}
$$

where $h_{\epsilon}$ is the impulse response corresponding to the transfer function

$$
H_{\epsilon} \triangleq \frac{\epsilon(s+\alpha-\epsilon)}{s(s+\alpha)}
$$

Remark that, for $\epsilon$ sufficiently small, the impulse response $h_{\epsilon}(\tau)$ is positive. This together with the nonpositivity of $g_{0}(\tau)$ readily implies that $\theta(\tau)$ is bounded above by a constant. Thus, all state variables are uniformly bounded. This in turn implies that they are uniformly continuous in $\tau$ since their derivatives with respect to $\tau$ are uniformly bounded, according to the error equations (5.8). This also implies that $g_{0}(\tau)$ is uniformly continuous.

Now (5.12) can also be rewritten as

$$
h_{\delta} * \int_{\tau_{r}}^{\tau} g_{0}(s) d s \geq \ln \left[\frac{\theta(\tau)}{\theta\left(\tau_{r}\right)}\right]-c \epsilon^{2} \geq-c
$$

where $h_{\delta}$ is the impulse response of $H_{\delta}(s) \triangleq \epsilon \frac{(s+\alpha-\epsilon)}{(s+\alpha)}$, and we have used the property of boundedness of $\theta(\tau)$ to establish the second inequality.

Assume $\alpha>\epsilon$, so that $H_{\delta}(s)$ is minimum-phase. Since $g_{0}(\tau) \leq 0$, the integral $\int g_{0}$ is nonincreasing and, from the minimum-phase assumption, it must be bounded below by a constant. ${ }^{15}$ Since the integrand is uniformly continuous and the integral is nonincreasing, the integrand must tend to zero, by Barbalat's lemma. In turn, this implies that $\lim _{\tau \rightarrow \infty} g_{0}(\tau)=0$. We will now prove that this leads to a contradiction.

From the definition of $g_{0}(\tau)(5.7)$ we see that the above limit is possible only if $\lim _{\tau \rightarrow \infty} z_{1}^{0}(\tau)=0$. However, one has

$$
z_{1}^{0}(\tau)=a(\theta) \theta^{2} \sin \left[\theta_{*} t(\tau)+\phi(\theta(\tau))\right]
$$

Since we have assumed $\theta>\theta_{*}$, then $a(\theta) \theta^{2}(\tau) \geq c>0,0>\phi(\theta(\tau))>-\pi, \forall \tau$. Then, the zero limit of $z_{1}^{0}(\tau)$ implies

$$
\lim _{\tau \rightarrow \infty} \sin \left[\theta_{*} t(\tau)+\phi(\theta(\tau))\right]=0
$$

Thus, an absurd results and therefore $\theta$ must arbitrarily approach $\theta_{*}$ for some large enough $\tau_{c}>\tau_{r}$.

\footnotetext{
${ }^{14}$ This does not mean that $\theta(\tau)$ will converge, or even remain close to $\theta_{*}$ for all $\tau>\tau_{c}$. The former will be implied because the state trajectory will then be "trapped" in the domain of exponential stability $\mathcal{D}$.

${ }^{15}$ Indeed, defining $v \triangleq h_{\delta} * \int_{\tau_{r}}^{\tau} g_{0}$, we also have $\int_{\tau_{r}}^{\tau} g_{0}=\bar{h}_{\delta} * v$, where $\mathcal{L} \bar{h}_{\delta}=(s+\alpha) /(s+\alpha-\epsilon)$. Thus, if $v \geq-c$ for some constant $c>0$, the same holds for $\int g_{0}$, provided $\alpha-\epsilon>0$.
} 
The case $\theta\left(\tau_{r}\right)<\theta_{*}$ is analyzed in analogous way. We first conclude that $\theta(\tau)$ is bounded below by a positive constant (which may depend on the initial conditions). The remainder follows exactly as in the preceding case. Note that the relevant property of $g_{0}(\tau)$ in this case is $0<\theta(\tau)<\theta_{*} \Rightarrow g_{0}(\tau)>0$.

\subsection{Convergence and exponential stability}

From Proposition 5.2, all trajectories enter a small compact set $\mathcal{D}_{0}=\left\{(y, \theta):\|y\| \leq \epsilon ;\left|\theta-\theta_{*}\right| \leq \epsilon\right\}$ at some finite time $t_{c}=t\left(\tau_{c}\right)$, provided $\epsilon$ is sufficiently small.

Now note that system (5.8), (5.11) is in the separated time scales standard form of averaging theory ([27], Section 4.4.1) (5.1-5.2). The latter is shown loc. cit. to be reducible to the conventional standard form of averaging theory $\dot{x}=\epsilon f(t, x)$. One can show that the averaged system is exponentially stable around the periodic orbit as in [2]. The same holds for the original system and the domain of exponential stability $\mathcal{D}$ around the equilibrium point $(y, \theta)=\left(0, \theta_{*}\right)$ is independent of $\epsilon$, for $\epsilon$ sufficiently small ([13], Theorem 7.5). Thus one can choose $\epsilon$ so that $\mathcal{D}_{0} \in \mathcal{D}$. This implies that the trajectories in the error space will eventually enter the domain $\mathcal{D}$ and exponential convergence will ensue.

\section{Tuning of the filter parameters}

The ANF contains two design parameters, the adaptation gain that determines the speed of adaptation, -hence its alertness to track frequency variations-, and the damping coefficient that determines the "depth of the notch", and consequently its noise sensitivity. A further contribution of our paper, which is presented in this section is the development of a tuning procedure to trade-off between adaptation alertness and noise sensitivity, preserving (exponential) stability. Of course, the procedure will be practically useful only if it is independent of the unknown $\theta_{*}$ and robust vis à vis $k$.

To underscore the importance of suitable tuning we first show that, even close to the equlibria, the ANF (both Regalia's and the one proposed here) can exhibit very complex dynamic behaviour if the tuning parameters are not well chosen. This will be done by proving that around the desired orbit the ANF is described by two coupled Hill's equations, one of them being a Mathieu equation, whose complexity of the stability-instability domains is well known [18]. A corollary of this study is the definition of a simple condition for the parameters which is "almost necessary" to avoid instability.

\subsection{Complex dynamics}

We are now only interested in the local behavior around the periodic orbit with $\theta=\theta_{*}>0$. As will be seen in section 6.3 , the normalization factor only changes gain $\gamma$ by a constant factor in such local analysis. Hence, we start our analysis without taking into account the normalization. Further, to reveal the complex dynamics we will work in the $\tau$ time-scale, which locally is always well defined.

From proposition 4.1 we know that the dynamics of the scaled ANF (3.1), (3.2), in the $\tau$ time scale, is described by (4.4)-(4.6). We are interested in the behaviour of this system close to the trajectory (4.9). We find convenient to introduce a change of coordinates $x_{3}(\tau)=\log (\theta(\tau))$. Taking the Jacobian of (the state realization of) (4.4)-(4.6) and evaluating it along the trajectory above gives the tangent approximation

$$
\dot{e}(\tau)=\left[\begin{array}{cccc}
0 & 1 & 0 & 0 \\
-1 & -2 \zeta+\frac{k^{2} \gamma}{2 \zeta} \sin (\tau) \cos (\tau) & 0 & \Psi(\tau) \\
0 & -k \gamma \cos (\tau) & 0 & \frac{k^{2} \gamma \theta_{*}}{2 \zeta} \cos ^{2}(\tau) \\
0 & 0 & \frac{-1}{\theta_{*}} & 0
\end{array}\right] e(\tau)
$$

where

$$
\Psi(\tau) \triangleq \theta_{*} k \cos (\tau)-\frac{\gamma \theta_{*} k^{3}}{4 \zeta^{2}} \cos ^{2}(\tau) \sin (\tau)
$$


We will now write this system as two coupled Hill's equations. The first two equations of (6.1) can be equivalently rewritten as

$$
e_{1}^{\prime \prime}(\tau)+\left[2 \zeta-\frac{k^{2} \gamma}{2 \zeta} \sin (\tau) \cos (\tau)\right] e_{1}^{\prime}(\tau)+e_{1}(\tau)=\Psi(\tau) e_{4}
$$

Also, differentiating one more time the last equation of (6.1) and replacing the third one we get

$$
e_{4}^{\prime \prime}(\tau)+\frac{k^{2} \gamma}{2 \zeta} \cos ^{2}(\tau) e_{4}(\tau)=\frac{k \gamma}{\theta_{*}} e_{1}^{\prime}(\tau)
$$

What we want to stress here is that the unforced part of (6.2) is a Mathieu equation of the form

$$
w^{\prime \prime}(\tau)-\frac{k^{2} \gamma}{4 \zeta}(1+\cos (2 \tau)) w(\tau)=0
$$

It is well known that the stability-instability boundaries of this equation are very complex, see e.g. [18]. In particular, it is known that the neighborhood around $\frac{k^{2} \gamma}{4 \zeta}=1$ is unstable. Therefore, an "almost" necessary condition for stability of our ANF is

$$
\frac{k^{2} \gamma}{4 \zeta}<1
$$

The sharpness of the above stability condition was tested by simulation as follows. We simulated both, the full scaled ANF (3.1), (3.2) and its tangent approximation (in the $\tau$ time scale) (6.1). In all simulations we set $k=1$, and for each $\zeta>0$, the adaptation gain $\gamma$ was increased until the stability limit was reached. The resulting stability boundaries, which may be found in [5], are surprisingly close.

\section{Remark 6.1}

It is of course possible, though highly improbable, that the couplings between the Hill's equations have a stabilizing effect. This is indeed contradicted by the simulations presented above.

\section{Remark 6.2}

The same analysis applied to Regalia's ANF (2.1), (2.2) leads to similar conclusions. In particular, it can be shown that $e_{4}(\tau)$ satisfies a damped Mathieu equation of the form

$$
e_{4}^{\prime \prime}(\tau)-\frac{k^{2} \gamma}{2 \zeta \theta_{*}^{2}} \sin (2 \tau) e_{4}^{\prime}(\tau)+\frac{k^{2} \gamma}{2 \zeta \theta_{*}^{4}} \cos ^{2}(\tau) e_{4}=\frac{k \gamma}{\theta_{*}^{3}} e_{1}^{\prime}(\tau)
$$

whose behaviour is even more complicated than the undamped one.

\subsection{A tuning procedure for exponential stability}

We will show in this subsection that an important advantage of introducing the scaling is that, for each given noise amplitude $k$, there is a simple procedure to tune the algorithm gains $\zeta$ and $\gamma$. We will see in the next subsection how normalization can further relax the requirement of knowledge of $k$. The analysis in this subsection is done in the $t$ time scale.

Before presenting this result we note that the periodic orbit for the scaled ANF (3.1), (3.2) is

$$
\left[\begin{array}{c}
\bar{x} \\
\overline{\bar{x}} \\
\bar{\theta}
\end{array}\right]=\left[\begin{array}{c}
\frac{-k}{2 \zeta} \cos \left(\theta_{*} t\right) \\
\frac{k \theta_{*}}{2 \zeta} \sin \left(\theta_{*} t\right) \\
\theta_{*}
\end{array}\right]
$$

\section{Proposition 6.1}

Consider the linear periodically time-varying three dimensional system

$$
\dot{z}=\left[\begin{array}{ccc}
0 & 1 & 0 \\
-1 & -2 \zeta & \sin t+\frac{1}{\zeta} \cos t \\
0 & -k^{2} \gamma \cos t & \frac{k^{2} \gamma}{2 \zeta} \cos t \sin t
\end{array}\right] z
$$

If the trivial equilibrium of (6.5) is exponentially stable, then (6.4) is an exponentially stable periodic orbit of the proposed scaled ANF (3.1), (3.2). 


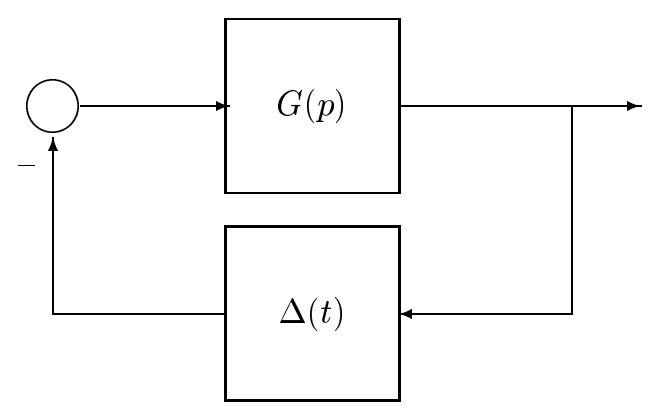

Figure 17:

\section{Proof}

The proof is based on Lyapunov's first method as applied to the linearization, along the orbit (6.4), of (3.1), (3.2). This procedure yields the linear periodically time-varying tangent approximation

$$
\dot{e}=\left[\begin{array}{ccc}
0 & 1 & 0 \\
-\theta_{*}^{2} & -2 \zeta \theta_{*} & k \theta_{*}\left[\sin \theta_{*} t+\frac{1}{\zeta} \cos \theta_{*} t\right] \\
0 & -k \gamma \theta_{*} \cos \theta_{*} t & \frac{k^{2} \gamma \theta_{*}}{2 \zeta} \cos \theta_{*} t \sin \theta_{*} t
\end{array}\right] e
$$

where $e$ is the deviation of the state with respect to the desired orbit. The proof is completed introducing the change of coordinates

$$
z=\left[\begin{array}{ccc}
1 & 0 & 0 \\
0 & \frac{1}{\theta_{*}} & 0 \\
0 & 0 & \frac{k}{\theta_{*}}
\end{array}\right] e
$$

and the time scale change $\frac{d s}{d t}=\theta_{*}$, which yields (6.5).

Even though a simple numerical study of (6.5) would provide us with some simple tuning rules, it is of course interesting to devise a more sistematic procedure to check stability of the scaled ANF. The study of the stability of linear periodically time-varying systems like (6.5) has a very long history, see e.g., [30], [26] and references therein. We present below an "absolute stability-like" sufficient condition where the search of the multipliers can be formulated as a convex optimization problem involving linear matrix inequalities $\dot{a}$ la [17]. For the sake of brevity we only recast the system of proposition 6.1 into this framework. An explicit stability condition, in terms of the tuning parameters $\gamma$ and $\zeta$, will be reported elsewhere.

\section{Corollary 6.1}

Consider the feedback system of Fig. 17 where $G(s)$ is a $2 \times 3$ transfer matrix with state space realization

$$
A \triangleq\left[\begin{array}{cccc}
0 & 1 & 0 & 0 \\
-1 & -2 \zeta & \frac{1}{\zeta} & 1 \\
0 & -\frac{k^{2} \gamma}{2} & 0 & -1 \\
0 & 0 & 1 & 0
\end{array}\right] ; B \triangleq\left[\begin{array}{cc}
0 & 0 \\
0 & 0 \\
1 & 0 \\
0 & 1
\end{array}\right] ; C \triangleq\left[\begin{array}{cccc}
0 & 2 \zeta & 0 & 0 \\
0 & 0 & 1 & 0 \\
0 & 0 & 0 & 1
\end{array}\right]
$$

which is asymptotically stable for all $\gamma, \zeta, k>0$, and $\Delta(t)$ is a periodically time-varying matrix

$$
\Delta(t) \triangleq \frac{k^{2} \gamma}{4 \zeta}\left[\begin{array}{ccc}
-\cos 2 t & \sin 2 t & 0 \\
-\sin 2 t & 0 & \sin 2 t
\end{array}\right]
$$

If this system is $\mathcal{L}_{2}$ stable, then (6.4) is an exponentially stable periodic orbit of the proposed scaled ANF (3.1), (3.2). 
Proof

First, we rewritte the linearized dynamics (6.5) as

$$
\dot{z}_{a}=A_{a}(t) z_{a} \triangleq\left[\begin{array}{cccc}
0 & 1 & 0 & 0 \\
-1 & -2 \zeta & \frac{1}{\zeta} & 1 \\
0 & -k^{2} \gamma \cos ^{2} t & \frac{k^{2} \gamma}{2 \zeta} \cos t \sin t & -1 \\
0 & -k^{2} \gamma \cos t \sin t & 1 & \frac{k^{2} \gamma}{2 \zeta} \cos t \sin t
\end{array}\right] z_{a}
$$

where we have defined $z_{a} \triangleq\left[z_{1}, z_{2}, z_{3} \cos t, z_{3} \sin t\right]^{T}$. The interest of this "system inmersion" is that the averaged system yields

$$
\frac{1}{\pi} \int_{0}^{\pi} A_{a}(t) d t \equiv A
$$

To prove that $A$ is exponentially stable for all $\gamma, \zeta, k>0$ we can do a root-locus analysis of the characteristic polynomial, which is given by

$$
1+\frac{k^{2} \gamma}{2 \zeta} \frac{s(s+\zeta)}{\left(s^{2}+2 \zeta s+1\right)\left(s^{2}+1\right)}=0
$$

The proof is completed checking that we can write these equations in the perturbation form

$$
\dot{z}_{a}=[A-B \Delta(t) C] z_{a}
$$

\section{Remark 6.3}

The importance of proposition 6.1 (and its corresponding corollary) stems from the fact that the system (6.5) is independent of $\theta_{*}$, hence for a given noise amplitude $k$, we can tune the gains $\gamma$ and $\zeta$ off-line. We should underscore that the reasoning above can not be used to derive a similar tuning procedure for the basic Regalia's ANF. This may be seen as follows. The Jacobian matrix of (the state realization $\left[x, \dot{x}, x_{3}\right]$ of) (2.1), (2.2) evaluated along the orbit (2.3) yields

$$
F\left(\theta_{*}, \theta_{*} t\right) \triangleq\left[\begin{array}{ccc}
0 & 1 & 0 \\
-\theta_{*}^{2} & -2 \zeta \theta_{*} & \frac{k}{\theta_{*}}\left[-\sin \left(\theta_{*} t\right)+\frac{1}{\zeta} \cos \left(\theta_{*} t\right)\right] \\
0 & -\frac{k \gamma}{\theta_{*}} \cos \left(\theta_{*} t\right) & \frac{k^{2} \gamma}{2 \zeta \theta_{*}^{3}} \cos \left(\theta_{*} t\right) \sin \left(\theta_{*} t\right)
\end{array}\right]
$$

We look now for a (linear) change of coordinates that transforms this matrix into a form $\theta_{*} E\left(\theta_{*} t\right)$, where the dependence of $E$ on $\theta_{*}$ is only through the sin and $\cos$ functions. If we can find such a matrix, the time scale change $\frac{d \tau}{d t}=\theta_{*}$ will finally yield a linear system independent of $\theta_{*}$, as done in the proof of proposition 6.1. But the existence of such a transformation implies the existence of a matrix $T(m)$ such that

$$
T(m)\left[\begin{array}{ccc}
0 & 1 & 0 \\
c_{1} m^{2} & c_{2} m^{2} & \frac{c_{3}}{m} \\
0 & \frac{c_{4}}{m} & \frac{c_{5}}{m^{3}}
\end{array}\right] T^{-1}(m)=m E_{0}
$$

with $E_{0}$ a matrix independent of $m$ and $c_{i}$ some constants. Since the trace is invariant under similarity transformation this woud imply the existence of another constant $c_{6} \quad\left(=\operatorname{trace}\left(E_{0}\right)\right)$ such that $c_{6} m=$ $c_{2} m^{2}+\frac{c_{5}}{m^{3}}$, which clearly is not possible for all $m$.

\subsection{Robust tuning procedure}

We will show in this subsection that for the scaled normalized ANF we can obtain a tuning procedure which, besides being independent of the unknown frequency $\theta_{*}$ as the tuning procedure above, is robust with respect to the noise amplitude $k$.

To this end, consider the linearized system (6.5) and notice the factor $\epsilon^{\prime} \triangleq k^{2} \gamma$. It is possible to regard this factor as a single design parameter. By direct application of averaging theory, one can assert that, for sufficiently small $\epsilon^{\prime}$, the linearized system is exponentially stable. Thus, there exists for each damping 
factor $\zeta$ a positive scalar $\epsilon_{1}^{\prime}(\zeta)$, such that the linearized system, and thus the periodic orbit, is (locally) exponentially stable $\forall \epsilon^{\prime} \in\left(0, \epsilon_{1}^{\prime}(\zeta)\right)$.

However, since we are dealing with external disturbances, the noise amplitude can change. Then, it would be eventually necessary to adjust $\gamma$ in order to keep the performance (or the stability) of the adaptive filter. Normalization circumvents this problem as follows. It can be easily verified that the inclusion of the normalization (4.12) does not affect neither the main orbit nor the linearized system around this orbit, except that $\epsilon^{\prime}=k^{2} \gamma$ must be replaced by

$$
\epsilon_{n o r}^{\prime} \triangleq \frac{k^{2} \epsilon}{\left(1+\frac{N k^{2}}{4 \zeta^{2}}\right)\left(1+\mu\left|\theta_{*}\right|^{\alpha}\right)}
$$

The expression above follows from the fact that on the orbit (6.4), one has

$$
\bar{x}^{2}+\left(\frac{\dot{\bar{x}}}{\theta_{*}}\right)^{2}=\frac{k^{2}}{4 \zeta^{2}}
$$

We see that the effective gain is now bounded $\forall k$ since $\epsilon_{n o r}^{\prime}<\frac{4 \zeta^{2} \epsilon}{N}$.

Local stability is therefore guaranteed for arbitrary noise amplitude if

$$
\frac{\epsilon}{N} \leq \frac{\epsilon_{1}^{\prime}(\zeta)}{4 \zeta^{2}}
$$

An approximate explicit local stability condition can be obtained from $(6.3)$, which gives $\epsilon_{1}^{\prime}(\zeta) \simeq 4 \zeta$. Thus, the condition is simply

$$
\frac{\epsilon}{N} \leq \frac{1}{\zeta}
$$

\section{Remark 6.4}

The above approximate expression for local stability shows that higher gain $\epsilon$ is allowed for smaller $\zeta$. This was confirmed by extensive simulations performed with the linearized system (6.5) modified with the normalized steady state gain according to (4.12), (6.7), see [5].

\subsection{Simulations}

The unstable behavior of Regalia's ANF due to inadequate tuning is shown in Fig. 18 and Fig. 19, where we repeated the simulations of Figs. $1-3$, but with $\gamma=4$ and $\zeta=5$, respectively.

Next, we illustrate the influence of the noise amplitude. The scaled but non-normalized ANF system with $\epsilon=1.35, \zeta=0.4, \theta_{*}=5, k=1$, IC : [1,1,10], results in fast and smooth response. However, with $k=10$ the system blows away. This means that the scaled ANF should be retuned according to the noise amplitude. Of course this is undesirable in practice. In contrast, normalization makes the ANF stability immune to noise amplitude changes. With $\mu=0.5 ; \alpha=2 ; N=1$ and $k=1000$ we have obtained Figs. 20-22. Stable behavior was observed for $k$ ranging from 1 to 1000.

As observed in Remark 6.4 normalization allows us to trade-off a higher gain $\epsilon$ with a smaller $\zeta$. To illustrate this point the simulations above (with $k=10$ ) were repeated for the normalized and scaled ANF and it was observed that stability was preserved for $\epsilon$ as high as 200. However, when the normalization was removed, $\epsilon$ had to be smaller than 0.012 to preserve stability. See [5].

\section{Conclusions}

We have solved in this paper the problem of global frequency estimation. The proposed algorithm is a scaled and normalized ANF inspired from the one reported in [23]. The new ANF was analyzed in terms of its stability, convergence and tuning. Despite a quite formidable complexity of its dynamical behavior, some conclusive results were established. A simple, yet sharp, rule for guaranteeing local stability was determined. The following fundamental and useful properties were established: (a) the new filter is shown to preserve 


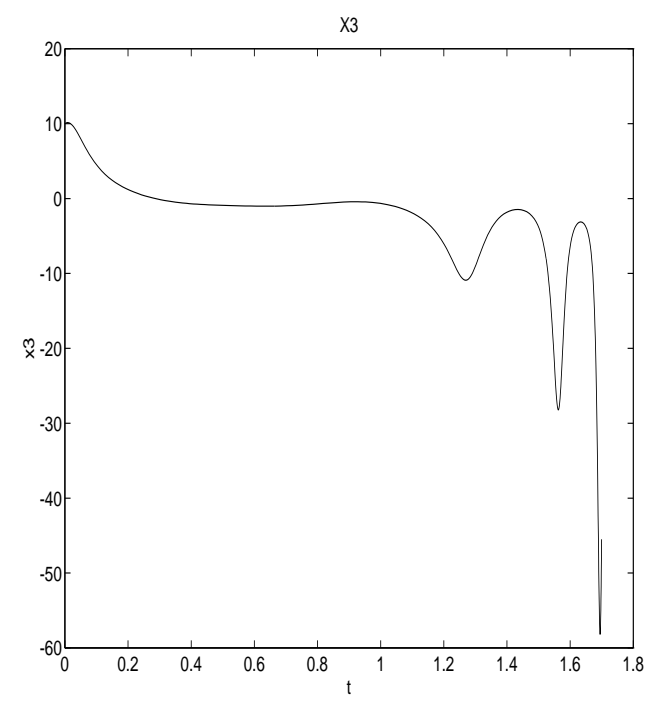

Figure 18: $\theta(t)$ (large $\gamma=4)$

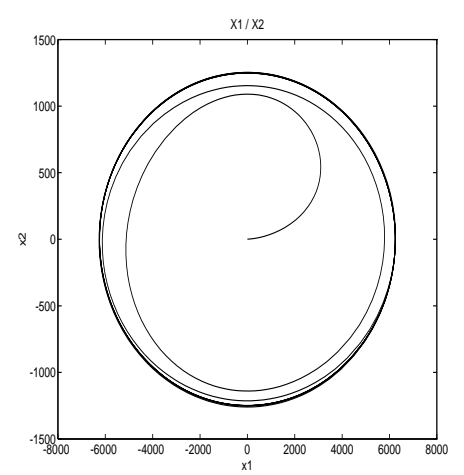

Figure 20: $\quad\left(x_{1}, x_{2}\right) \quad$ (stability with arbitrary $k$ )

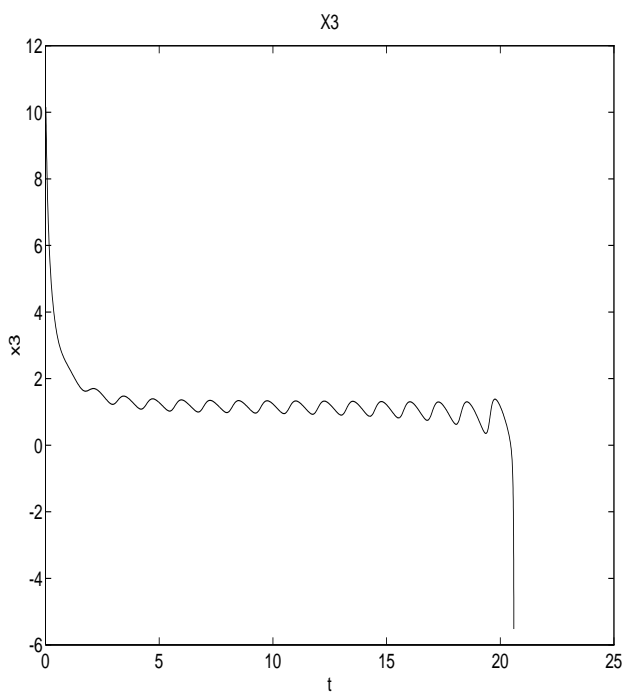

Figure 19: $\theta(t)$ (large $\zeta=5)$

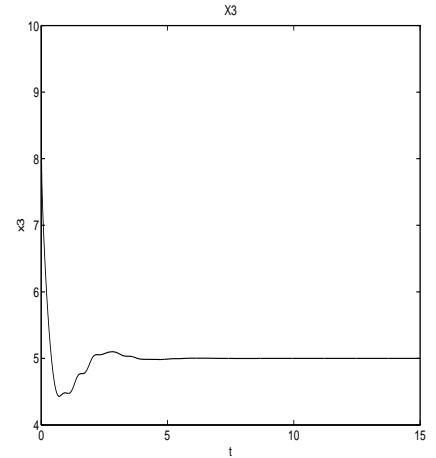

Figure 21: $\theta(t)$

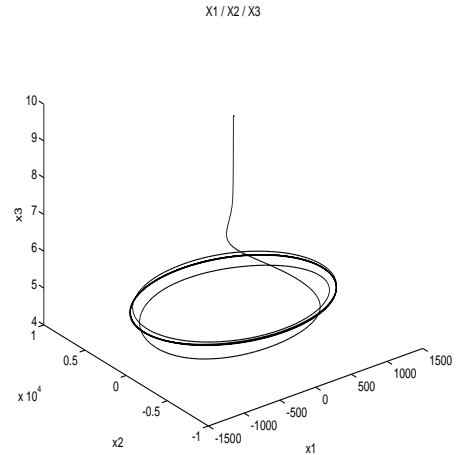

Figure 22: 3D state-space plot

local exponential stability under arbitrary frequency changes of the sinusoidal noise, thanks to scaling; (b) normalization also allows local stability preservation for arbitrary changes of the noise amplitude; (c) the new filter can be made globally asymptotically stable under sufficiently small adaptation gain. Neither of the latter mentioned properties are encountered in the original version of the ANF.

Even though we concentrated our attention here in the simplest case of a single constant frequency without noise, in [5] we have shown, -via simulations and some analysis,- that the algorithm is able to track time-varying frequencies, preserves local stability in the presence of multiple sinusoids, and is robust vis $\dot{a}$ vis noise.

Some preliminary results about the integration of the new adaptive notch filter in noise cancellation systems, in the spirit of [2], are reported in [5]. Also, current research is under way to modify the filter to estimate multiple frequencies. The outcome of this research will be reported elsewhere.

\section{Acknowledgments}

Part of this work was realized while the second author was visiting Prof Hsu in the University of Rio de Janeiro. The warm hospitality of this group is deeply acknowledged. Also, the second author would like to thank Anders Rantzer for suggesting the change of coordinates used in the proof of corollary 6.1. The work of Gilney Damm in LSS is sponsored by the brazilian foundation CAPES. 


\section{References}

[1] S. Bittanti, M. Campi and S. Savaresi, Unbiased estimation of a sinusoid in colored noise via adapted notch filters, Automatica, (to appear).

[2] M. Bodson and S. Douglas, Adaptive algorithms for the rejection of sinusoidal disturbances with unknown frequency, Prof 13th IFAC World Conf., San Francisco, CA, USA, July 1-5, 1996.

[3] E. A. Coddington and N. Levinson, Theory of Ordinary Differential Equations, McGraw-Hill, New York, 1955.

[4] C. Fuller and A. von Flotow, Active control of sound and vibration, IEEE Cont. Syst. Magazine, vol. 15, no. 6, Dec. 1996, pp. 9-19.

[5] G. R. Damm, Application of a global frequency estimator to sinusoidal noise cancellation. M. Sc. Thesis, COPPE/U. Fed. de Rio de Janeiro, Brazil, Sept. 1997. (In portuguese)

[6] C. Desoer and M. Vidyasagar, Feedback Systems: Input-Output Properties, Ac. Press, NY, 197

[7] B. Friedlander and O. J. Smith, Analysis and performance evaluation of an adaptive notch filter, IEEE Trans. on Inf. Th., Vol. 30, pp. 283-295, 1984.

[8] P. Handel, Estimation methods for narrow band signals, Ph D Thesis, Uppsala, Sweden, 1993.

[9] S. Hall and N. Wereley, Performance of higher harmonic control algorithms for helicopter vibration reduction, J. Guidance Control and Dynamics, vol. 116, no. 4, pp. 793-797, 1993.

[10] R. Herzog, P. Buhler, C. Gahler and R. Larsonneur, Unbalance compensation using generalized notch filters in the multivariable feedback of magnetic bearings, IEEE Trans. Control Syst. Techn., vol. 4, no. 5, pp. 580-586, sept. 1996.

[11] U. Jonsson and A. Rantzer, Systems with uncertain parameters- time-variations with bounded derivatives, Prof 13th IFAC World Cof., San Francisco, CA, USA, July 1-5, 1996.

[12] S. M. Kay and S. L. Marple, Spectrum analysis - a modern perspective, Proc. IEEE, Vol. 69, pp. 1380-1419, 1981.

[13] H. Khalil, Nonlinear systems, 2nd. ed., Prentice Hall, NJ, 1996.

[14] L. Ljung, System Identification: Theory for the User, Englewood Cliffs, NJ, Prentice-Hall, 1987.

[15] A. M. Lyapunov, Stability of Motion, Math. in Science and Engineering, vol. 30, Academic Press, NY, 1966.

[16] I. Mareels and J. W. Polderman, Adaptive systems: An introduction, Birkhauser, 1996.

[17] A. Megretski and A. Rantzer, System analysis via integral quadratic constrained, IEEE Trans. Automat. Contr., vol. 42, no. 7, June 1997.

[18] K. Narendra and J. Taylor, Frequency domain criteria for absolute stability, New York: Academic Press, 1973.

[19] A. Nehorai, A minimal parameter adaptive notch filter with constrainde poles and zeros, IEEE Trans. Accoustics, Speech and Signal Processing, vol. 33, pp. 983-996, 1985.

[20] R. Ortega, On Morse's New Adaptive Controller: Parameter Convergence and Transient Performance, IEEE Trans. Aut. Cont., Vol. AC-38, No. 8, pp. 1191-1202, Aug. 1993.

[21] V. F. Pisarenko, The retrieval of harmonics from a covariance function, Geophys. J. Royal Astron. Sc., Vol. 33, pp. 347-366, 1973.

[22] V. Reddy, B. Egardt and T. Kailath, Least-squares type algorithm for adaptive implementation of Pisarenko's harmonic retrieval method, IEEE Trans. Accoustics, Speech and Signal Processing, vol. 30, pp. 399-405, June 1982. 
[23] P. Regalia, An improved lattice-based adaptive IIR notch filter, IEEE Trans. on Signal Proc., vol. 39, no. 9, pp. 2124-2128, 1991.

[24] P. Regalia, IIR filtering in signal processing and control, Marcel Dekker, New York, 1995.

[25] B. Riedle and P. Kokotovic, Integral manifolds of slow adaptation, IEEE Trans. Automat. Contr., Vol. AC-31, No. 4, pp. 316-324, April 1986.

[26] W. Rugh, Linear system theory, Prentice Hall, NJ, 2nd. ed., 1996.

[27] S. Sastry and M. Bodson, Adaptive control - stability, convergence and robustness, Prentice-Hall, New Jersey, 1989.

[28] J. Treichler, Transient and convergent behavior of the adaptive line enhancer, IEEE Trans. Accoustics, Speech and Signal Processing, vol. 27, pp. 53-63, Feb 1979.

[29] B. Widrow, et al., Adaptive noise cancelling: Principles and applications, Proc. IEEE, Vol. 63, pp. 1692-1716, Dec. 1975.

[30] V. Yakubovich and V. Starzhinkii, Parametric resonance in linear systems, Izd. Nauka, Mow., 1987. (In russian).

\section{A Boundedness of $g_{0}, g_{1}, g_{2}$ and $\kappa$}

Consider the expressions for $g_{0}, g_{1}, g_{2}$ given in (5.7) and the expressions for $\gamma$ and $z_{1}^{0}$ given by (4.16) and (5.3). Note also that $x^{\prime}=\dot{x} \theta^{-1}=z_{2}$ and $\rho_{z}^{2}=z_{1}^{2}+z_{2}^{2} \geq z_{2}^{2}$. Then, we easily conclude that $\exists c>0$ such that

$$
g_{0} \leq k^{2} c ; g_{1} \leq c ; g_{2} \leq k c
$$

As for $\kappa$, it follows from (4.8) that

$$
|\kappa|<\frac{2 \zeta \rho_{z}^{2}}{1+N \rho_{z}^{2}}+\frac{k \rho_{z}}{1+N \rho_{z}^{2}} \leq c(1+k)
$$

where, $\rho_{z}:=\|z\|$. The existence of a constant $c$ follows from the fact that both fractions in (A.1) are analytic $\forall \rho_{z} \in \Re$ and have finite limits for $\rho \rightarrow 0$ or $\infty$. In fact, the evaluation of the maximum value of these fractions results in the bound (4.20).

\section{B Boundedness of $b_{1}$}

For fixed $k, N$ and $\zeta$, we have the following bounds:

$$
\begin{aligned}
\left|\theta\left(\partial z_{1}^{0} / \partial \theta\right)\right| & \leq k c \\
\left|\left[z_{2}^{0}+\theta\left(\partial z_{2}^{0} / \partial \theta\right)\right]\right| & \leq k c
\end{aligned}
$$

For convenience we denote by $P_{i}\left(\theta_{r} ; t\right)$ a generic $i$-th order polynomial of $\theta_{r} \triangleq \theta / \theta_{*}$ with time dependent coefficients. In our particular problem, the coefficients are periodic in time. If the polynomial has constant coefficients, then we denote it by $P_{i}\left(\theta_{r}\right)$.

The bound (B.1) is obtained as follows. Let

$$
F_{1} \triangleq \theta\left(\partial z_{1}^{0} / \partial \theta\right)
$$

It follows from (5.3) that

$$
z_{1}^{0}=k \theta_{r}^{2} P_{2}\left(\theta_{r} ; t\right) / P_{4}\left(\theta_{r}\right)
$$

Then, we have

$$
\partial z_{1}^{0} / \partial \theta=k \theta_{r} P_{6}\left(\theta_{r} ; t\right) / P_{8}\left(\theta_{r}\right)
$$




$$
\theta\left(\partial z_{1}^{0} / \partial \theta\right)=k \theta_{r}^{2} P_{6}\left(\theta_{r} ; t\right) / P_{8}\left(\theta_{r}\right)
$$

Since $P_{8}$ in the above expression is given by $P_{8}=\left[\left(\theta_{r}^{2}-1\right)^{2}+4 \zeta^{2} \theta_{r}^{2}\right]^{2}$, which does not have real roots, it follows that $F_{1}$ is analytic $\forall \theta \in \Re$. As before, the limits for $\theta_{r} \rightarrow 0$ or $\infty$ are bounded. Consequently, $F_{1}$ is uniformly bounded by a constant and thus (B.1) is obtained.

Finally, the bound (B.2) is obtained in similar way, using the following relationships derived from (5.4)

$$
\begin{gathered}
z_{2}^{0}=\theta^{-1} \frac{\partial z_{1}^{0}}{\partial t} \\
F_{2}:=\theta\left(\partial z_{2}^{0} / \partial \theta\right)=\theta \frac{\partial}{\partial \theta}\left(\theta^{-1} \partial z_{1}^{0} / \partial t\right)
\end{gathered}
$$

We can also write

$$
F_{2}=\left[-\theta^{-1} \frac{\partial z_{1}^{0}}{\partial t}+\frac{\partial}{\partial t} \frac{\partial z_{1}^{0}}{\partial \theta}\right]
$$

Then, from (B.5)(B.6), one has

$$
\begin{gathered}
z_{2}^{0}=k \theta_{r} P_{2}\left(\theta_{r} ; t\right) / P_{4}\left(\theta_{r}\right) \\
F_{2}=k \theta_{r}\left[P_{2}\left(\theta_{r} ; t\right) / P_{4}\left(\theta_{r}\right)+P_{6}\left(\theta_{r} ; t\right) / P_{8}\left(\theta_{r}\right)\right]
\end{gathered}
$$

Since the functions $F_{2}$ and $z_{2}^{0}$ are analytic $\forall \theta_{r}$ as before, and their limits for $\theta_{r} \rightarrow 0$ or $\infty$ are bounded, then these functions are also uniformly bounded by constants. Thus, the bound (B.2) results. 